\title{
Probabilistic constrained multicast beamforming in cognitive relay systems
}

\author{
Shengyu Li, Wenjun $\mathrm{Xu}^{*}$, Xiaomei Lu and Jiaru Lin
}

\begin{abstract}
In this paper, multicast beamforming in cognitive relay systems is investigated with the consideration of imperfect channel state information at the transmitter (CSIT). We focus at the design of the optimal signal forwarding matrix at the cognitive relay in both centralized relay mode (CRM) and distributed relay mode (DRM). The problem is formulated aiming at minimizing the total consumed power at the relay node with suitable QoS guarantee for secondary users and strict interference control for primary users. Due to the uncertainty of transmission channel gains, constraints for QoS guarantee and interference control cannot be expressed in closed forms, making it extremely difficult to solve the problem directly. To circumvent this, we first employ the Bernstein-type inequality to convert the probabilistic constraints into closed-form expressions and then present both the semi-definite relaxation (SDR) algorithm and the penalty function (PenFun) algorithm to accomplish the non-convex problem optimization. Simulation results show that CRM is more resource efficient than DRM, and the PenFun algorithm can achieve a much better solution than the SDR algorithm at the cost of complexity. Meanwhile, compared with existing schemes which do not consider the CSIT error, the proposed schemes can support a much lower outage probability and enjoy perfect interference control for primary users.
\end{abstract}

Keywords: Multicast beamforming; Cognitive relay systems; Imperfect channel state; Bernstein-type inequality; Penalty function method

\section{Introduction}

In recent years, along with the development of wireless communication technologies and the proliferation of multimedia services, multicast transmission has become an indispensable part of mobile communication systems, e.g., Multimedia Broadcast/Multicast Service (MBMS) in 3GPP [1], Broadcast and Multicast Service (BCMCS) in 3GPP2 [2], and Multicast and Broadcast Service (MBS) in IEEE 802.16 [3]. As a result, large amounts of effort has been made to design resource-efficient multicast schemes, among which cooperative multicast with the help of multi-antenna relay nodes has attracted many researchers' attention [4-7].

In the cooperative multicast scheme, multi-antenna relay nodes may operate in either the distributed or the centralized manner. When operating in the distributed manner, each antenna forwards its own received signal

*Correspondence: wjxu@bupt.edu.cn

Key Lab of Universal Wireless Communications, Ministry of Education, Beijing University of Posts and Telecommunications, Xitucheng Road, Haidian District, Beijing, China with a respective amplification factor. For example, aiming at minimizing the total transmit power, the authors in $[4,5]$ have studied the amplification factor optimization for a single multicast session and multiple multicast sessions, respectively. The model is superior when information exchange among different antennas is difficult, e.g., cooperative multicast in wireless sensor networks. By contrast, when the relay node operates in the centralized manner, each antenna forwards a combination of all received signals. In other words, joint signal processing across different antennas is executed in this manner. Although the implementation complexity increases, the supported throughput is much higher due to more optimization degrees of freedom [6,7].

Meanwhile, cognitive radio (CR), allowing secondary users to access the authorized spectrum bands with suitable interference control, is able to significantly improve the spectrum utilization and has been acknowledged as one of the most potential technologies in the next communication system [8]. Although cognitive beamforming has been well developed for several years, existing researches

\section{Springer}

(c) $2015 \mathrm{Li}$ et al: licensee Springer. This is an Open Access article distributed under the terms of the Creative Commons Attribution License (http://creativecommons.org/licenses/by/4.0), which permits unrestricted use, distribution, and reproduction in any medium, provided the original work is properly credited. 
mainly focus on beamformer design at cognitive base stations, either for secondary users' data transmission with restricted transmit power $[9,10]$ or for primary-secondary cooperation with careful designed cooperation protocols $[11,12]$. There are also some works paying attention to the combination of multicast transmission and CR technology [13-15]. However, as far as we know, most of those researches concentrate on one-hop transmission, with few papers concerning cooperative multicast, especially multicast transmission via multi-antenna relays.

In this paper, the CR technology and the cooperative multicast are jointly considered, and the multicast beamforming problem in cognitive relay systems is investigated. As mentioned above, the relay node can operate in either the centralized manner or the distributed manner, and these two relay modes, denoted as centralized relay mode (CRM) and distributed relay mode (DRM) respectively, have their own strong points and weakness. Hence, both CRM and DRM are included in our problem formulation. Furthermore, due to the channel feedback error and the feedback delay, perfect channel state information at the transmitter (CSIT) is always unavailable in practice. Unfortunately, to the best of our knowledge, most of the existing researches $[4-7,13-15]$ only apply to perfect CSIT feedback model. Therefore, imperfect CSIT feedback is also considered in our research model to enhance the practicability of our study.

More specially, we pay attention to the design of the optimal multicast signal forwarding matrix at the multiantenna cognitive relay. The formulated problem aims at minimizing the total consumed power at the relay node, subject to the constraints that the minimum received signal-to-interference-plus-noise ratio (SINR) requirement at each cognitive destination and the maximum received interference requirement at each primary user are satisfied with a prescribed, usually very high, probability. Different from the model of perfect CSIT feedback, constraints for the SINR guarantee and the interference control are in probabilistic forms, instead of closed forms. This characteristic makes it difficult to solve the formulated problem as we cannot compute the probability directly.

In this paper, a safe approximation is presented to solve the question, and detailed algorithms for both DRM and CRM are carefully designed. In DRM, the optimal forwarding matrix is a diagonal matrix. To optimize the diagonal vector, the Bernstein-type inequality is utilized to transform the probabilistic constraints into closed-form expressions, making the problem easy to tackle. Thereafter, the semi-definite relaxation (SDR) method and the penalty function (PenFun) method are adopted to solve the problem efficiently. In CRM, we first prove that the optimal forwarding matrix is the combination of a matched-filter receiver and an adaptive beamformer and then jointly exploit the Bernstein-type inequality and the PenFun method to accomplish the adaptive beamformer design.

\section{Notation}

Throughout this paper, the following notations are defined. $\mathbb{C}^{N \times M}$ and $\mathbb{D}^{N}$ denote the set of all $N \times M$ complex matrices and the set of all $N \times N$ diagonal complex matrices, respectively. $\mathbf{I}$ is the identity matrix with a suitable dimension. $\mathcal{C N}\left(\mu, \sigma^{2}\right)$ is the complex Gaussian distribution with mean value $\mu$ and variance $\sigma^{2}$, and $\operatorname{Pr}\{\theta\}$ is the probability that case $\theta$ occurs. $\mathbf{A} \succeq \mathbf{0}$ means that matrix $\mathbf{A}$ is positive semi-definite, and $\mathbf{A}^{\perp}$ is the orthonormal basis for the null space of $\mathbf{A} . \mathbf{A}^{H},\|\mathbf{A}\|, \operatorname{Re}[\mathbf{A}], \operatorname{Tr}[\mathbf{A}]$, $\operatorname{Rank}[\mathbf{A}]$ and $\lambda_{\max }(\mathbf{A})$ mean the conjugate transpose, the Frobenius norm, the real part, the trace, the rank and the maximal eigenvalue of matrix $\mathbf{A}$, respectively.

\section{System model and problem formulation}

We consider a cognitive relay system where a $N$-antenna cognitive relay forwards message transmitted by a cognitive source to $K$ cognitive destinations. Meanwhile, $L$ primary users are active and communicating on the same authorized spectrum band. Similar to [4-7], it is assumed that there is no direct link from the source node to destinations and the communication can only be completed via the relay. Figure 1 is a simple illustration of the considered cognitive relay system.

\subsection{Imperfect CSIT feedback model}

For the sake of convenience, some symbols are predefined here. $\mathbf{f} \in \mathbb{C}^{N \times 1}$ is the transmission channel vector from the cognitive source to the cognitive relay. $\mathbf{h}_{\mathrm{c}, k} \in \mathbb{C}^{N \times 1}$ and $\mathbf{h}_{\mathrm{p}, l} \in \mathbb{C}^{N \times 1}$ are the transmission channel vectors from the cognitive relay to destination $k$ and primary user $l$, respectively.

This paper concerns how to select the optimal forwarding matrix $\mathbf{W}$ at the cognitive relay (shown in the next subsection). Hence, $\mathbf{f}$ is assumed to be perfectly known since it can be obtained by channel estimation at the cognitive relay. By contrast, $\mathbf{h}_{\mathrm{c}, k}$ and $\mathbf{h}_{\mathrm{p}, l}$, which are obtained by channel feedback from cognitive destinations and primary users, are imperfectly known at the cognitive relay.

The imperfect CSIT feedback is modeled as $[16,17]$

$$
\begin{aligned}
& \mathbf{h}_{\mathrm{c}, k}=\hat{\mathbf{h}}_{\mathrm{c}, k}+\mathbf{e}_{\mathrm{c}, k}, k=1,2, \ldots, K, \\
& \mathbf{h}_{\mathrm{p}, l}=\hat{\mathbf{h}}_{\mathrm{p}, l}+\mathbf{e}_{\mathrm{p}, l}, l=1,2, \ldots, L,
\end{aligned}
$$

where $\hat{\mathbf{h}}_{\mathrm{c}, k}$ and $\hat{\mathbf{h}}_{\mathrm{p}, l}$ are the presumed CSIT at the cognitive relay for destination $k$ and primary user $l$, respectively, and $\mathbf{e}_{\mathrm{c}, k}$ and $\mathbf{e}_{\mathrm{p}, l}$ are the corresponding CSIT feedback errors.

Similar to [17], it is assumed that the CSIT feedback errors are complex Gaussian distributed, i.e., $\mathbf{e}_{\mathrm{c}, k} \sim$ $\mathcal{C N}\left(\mathbf{0}, \mathbf{C}_{\mathrm{c}, k}\right), \mathbf{e}_{\mathrm{p}, l} \sim \mathcal{C N}\left(\mathbf{0}, \mathbf{C}_{\mathrm{p}, l}\right)$. For brevity, $\mathbf{C}_{\mathrm{c}, k}$ and $\mathbf{C}_{\mathrm{p}, l}$ 


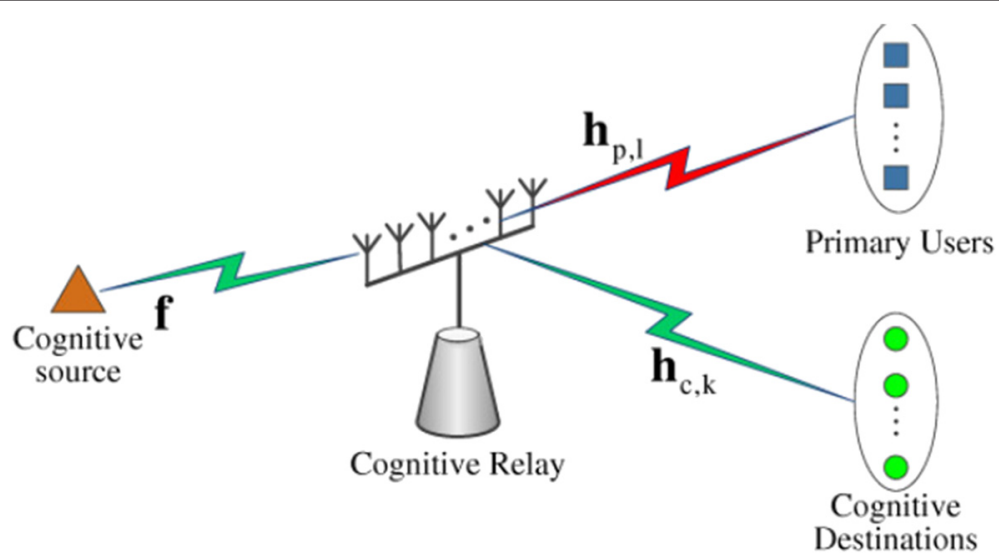

Figure 1 Illustration for multicast beamforming in cognitive relay systems. The cognitive relay forwards the message transmitted by the cognitive source to $K$ cognitive destinations by reusing the authorized spectrum of $L$ primary users. Antennas at the cognitive relay may be deployed either together or discretely, and this figure only shows the the case of together.

are simplified as $\mathbf{C}_{\mathrm{c}, k}=\sigma_{\mathrm{c}, k}^{2} \mathbf{I}, \mathbf{C}_{\mathrm{p}, l}=\sigma_{\mathrm{p}, l}^{2} \mathbf{I}$. This simplification is to facilitate our description below and does not change the overall analysis of our problem.

\subsection{Transmission model and problem formulation}

The total transmission process is divided into two slots. In the first slot, the cognitive source broadcasts message $x$ to the cognitive relay.

$$
\mathbf{y}_{\mathrm{R}}=\mathbf{f} x+\mathbf{z}_{\mathrm{R}}
$$

where $\mathbf{z}_{\mathrm{R}} \sim \mathcal{C N}\left(\mathbf{0}, \sigma_{z}^{2} \mathbf{I}\right)$ is the the received noise at the cognitive relay.

In the second slot, the cognitive relay forwards the received message $\mathbf{y}_{\mathrm{R}}$ to destinations with a forwarding matrix $\mathbf{W} \in \mathbb{C}^{N \times N}$. The forwarding signal is

$$
\mathbf{s}_{\mathrm{R}}=\mathbf{W} \mathbf{y}_{\mathrm{R}}=\mathbf{W} \mathbf{f} x+\mathbf{W} \mathbf{z}_{\mathrm{R}}
$$

and the final received signal at destination $k$ is

$$
y_{k}=\mathbf{h}_{\mathrm{c}, k}^{H} \mathbf{s}_{\mathrm{R}}+z_{k}=\mathbf{h}_{\mathrm{c}, k}^{H} \mathbf{W} \mathbf{f} x+\mathbf{h}_{\mathrm{c}, k}^{H} \mathbf{W} \mathbf{z}_{\mathrm{R}}+z_{k},
$$

where $z_{k} \sim \mathcal{C N}\left(0, \sigma_{z}^{2}\right)$ is the received interference plus noise.

Based on Equation 5, the final received SINR at destination $k$ could be calculated as

$$
\operatorname{SINR}_{k}=\frac{\left|\mathbf{h}_{\mathrm{c}, k}^{H} \mathbf{W} \mathbf{f} x\right|^{2}}{\left|\mathbf{h}_{\mathrm{c}, k}^{H} \mathbf{W} \mathbf{z}_{\mathrm{R}}\right|^{2}+\sigma_{z}^{2}}=\frac{p_{\mathrm{s}}\left|\mathbf{h}_{\mathrm{c}, k}^{H} \mathbf{W f}\right|^{2}}{\sigma_{z}^{2}\left\|\mathbf{h}_{\mathrm{c}, k}^{H} \mathbf{W}\right\|^{2}+\sigma_{z}^{2}},
$$

where $p_{\mathrm{S}}$ is the transmit power of the cognitive source.
Similarly, the total received interference from the signal forwarding at the cognitive relay node to primary user $l$ can be estimated as

$I_{l}=\left|\mathbf{h}_{\mathrm{p}, l}^{H} \mathbf{W} \mathbf{f} x\right|^{2}+\left|\mathbf{h}_{\mathrm{p}, l}^{H} \mathbf{W} \mathbf{z}_{\mathrm{R}}\right|^{2}=p_{\mathrm{s}}\left|\mathbf{h}_{\mathrm{p}, l}^{H} \mathbf{W} \mathbf{f}\right|^{2}+\sigma_{z}^{2}\left\|\mathbf{h}_{\mathrm{p}, l}^{H} \mathbf{W}\right\|^{2}$.

And the total consumed power at the cognitive relay is

$$
P_{\mathrm{R}}=\|\mathbf{W} \mathbf{f} x\|^{2}+\left\|\mathbf{W} \mathbf{z}_{\mathrm{R}}\right\|^{2}=p_{\mathrm{s}}\|\mathbf{W} \mathbf{f}\|^{2}+\sigma_{z}^{2}\|\mathbf{W}\|^{2} .
$$

In this paper, two kinds of relay modes at the relay node are adopted for cooperative multicast transmission [4-7], i.e.,

- Centralized relay mode (CRM): In this mode, joint signal processing is allowed. Therefore, each antenna forwards a combination of the received signals [7], and $\mathbf{W}$ can be chosen as any $N \times N$ complex matrix, i.e.,

$$
\mathbf{W} \in \mathbb{C}^{N \times N} .
$$

This mode is preferable when antennas are deployed together and information exchange is available.

- Distributed relay mode (DRM): In this mode, each antenna forwards its own received signal independently $[4,5]$, and as a result, $\mathbf{W}$ should be a diagonal matrix, i.e.,

$$
\mathbf{W}=\operatorname{Diag}(\mathbf{w}) \in \mathbb{D}^{N} .
$$

This mode is suited for the case where relay antennas are far apart from each other and large amounts of information exchange is difficult, except for channel state information.

Finally, the optimal forwarding matrix design is formulated as an optimization problem which aims at 
minimizing the total consumed power at the cognitive relay with prescribed transmission QoS guarantee for destination nodes and interference control for primary users.

$$
\begin{aligned}
\mathcal{Q}: \quad \min _{\mathbf{W}} P_{\mathrm{R}} \\
\mathcal{C}_{1}: \operatorname{Pr}\left\{\operatorname{SINR}_{k}<\gamma_{\text {th }}\right\} \leq \rho_{\mathrm{c}}, \forall k \\
\mathcal{C}_{2}: \operatorname{Pr}\left\{I_{l}>I_{\mathrm{th}, l}\right\} \leq \rho_{\mathrm{p}}, \forall l \\
\mathcal{C}_{3}: \operatorname{P}_{\mathrm{R}} \leq P_{\text {th }} \\
\mathcal{C}_{4}: \begin{cases}\mathbf{W} \in \mathbb{C}^{N \times N}, & \text { for CRM } \\
\mathbf{W} \in \mathbb{D}^{N}, & \text { for DRM }\end{cases}
\end{aligned}
$$

where $\gamma_{\text {th }}$ is the minimum required transmission SINR of the broadcast message, $I_{\mathrm{th}, l}$ is the interference threshold for primary user $l$, and $P_{\text {th }}$ is the maximum transmit power at the cognitive relay. $\mathcal{C}_{1}$ implies that the maximum outage probability for any cognitive destination should be no more than $\rho_{\mathrm{c}} . \mathcal{C}_{2}$ restricts that the probability that primary user l's received interference exceeds the prescribed threshold $I_{\mathrm{th}, l}$ should be less than $\rho_{\mathrm{p}}$. Throughout this paper, $\mathcal{C}_{1}$ and $\mathcal{C}_{2}$ are also named as the maximum outage probability constraint for each cognitive destination and the maximum over-interference probability constraint for each primary user for short.

\section{Remark 1.}

- To make a better understanding on these investigated two relay modes, we compare CRM and DRM to Joint Processing (JP) and Coordinated Beamforming $(C B)$ in Coordinated Multi-Point transmission (CoMP) [18], where transmit antennas in our model correspond to transmit points in CoMP. In JP, both transmission data and channel information need to be shared among different transmit points, and a joint signal processing is required, while in $C B$, only channel information exchange is necessary and each point optimizes its own beamformer in a distributed manner.

- Meanwhile, perfect synchronization among antennas is assumed in this paper, for both CRM and DRM. In CRM, synchronization could be simply achieved as antennas are deployed together. In DRM, although antennas are deployed apart from each other, a necessary backhaul link, either wired or wireless, is required to bear the channel information exchange, which could also be responsible for the antenna synchronization. This is similar to the case of $C B$, where a limited backhaul link is established to take charge of the channel information exchange and the point synchronization.

\section{Algorithm design for DRM}

In this section, the optimal forwarding matrix design problem for DRM is handled, which is indeed to optimize its diagonal vector. Toward this end, the Bernsteintype inequality and the SDR/PenFun method are jointly employed to solve the complex problem. In the next section, we prove that the optimal forwarding matrix W in CRM should be a rank-one matrix. Based on this observation, the optimization of the forwarding matrix is transformed into the beamformer design, which can also be efficiently solved with the PenFun method.

\subsection{Problem transformation}

As the forwarding matrix $\mathbf{W}=\operatorname{Diag}(\mathbf{w})$ is a diagonal matrix, the received signal at destination $k$ in DRM can be simplified as

$y_{k}=\mathbf{h}_{\mathrm{c}, k}^{H} \mathbf{W} \mathbf{f} x+\mathbf{h}_{\mathrm{c}, k}^{H} \mathbf{W} \mathbf{z}_{\mathrm{R}}+z_{k}=\mathbf{h}_{\mathrm{c}, k}^{H} \mathbf{F} \mathbf{w} x+\mathbf{h}_{\mathrm{c}, k}^{H} \mathbf{Z}_{\mathrm{R}} \mathbf{w}+z_{k}$,

where $\mathbf{F}=\operatorname{Diag}(\mathbf{f})$ and $\mathbf{Z}_{R}=\operatorname{Diag}\left(\mathbf{z}_{R}\right)$. Hence, the received SINR at destination $k$ in Equation 6 can be rewritten as

$$
\mathrm{SINR}_{k}^{\mathrm{DRM}}=\frac{\left|\mathbf{h}_{\mathrm{c}, k}^{H} \mathbf{F} \mathbf{w} x\right|^{2}}{\left|\mathbf{h}_{\mathrm{c}, k}^{H} \mathbf{Z}_{\mathrm{R}} \mathbf{w}\right|^{2}+\sigma_{z}^{2}}=\frac{p_{\mathrm{s}}\left|\mathbf{h}_{\mathrm{c}, k}^{H} \mathbf{F w}\right|^{2}}{\sigma_{z}^{2}\left|\mathbf{h}_{\mathrm{c}, k}^{H} \mathbf{w}\right|^{2}+\sigma_{z}^{2}}
$$

Likewise, the received interference at primary user $l$ can be rewritten as

$$
I_{l}^{\mathrm{DRM}}=p_{\mathrm{s}}\left|\mathbf{h}_{\mathrm{p}, l}^{H} \mathbf{F w}\right|^{2}+\sigma_{z}^{2}\left|\mathbf{h}_{\mathrm{p}, l}^{H} \mathbf{w}\right|^{2} .
$$

And the total consumed power at the cognitive relay is

$$
P_{\mathrm{R}}=p_{\mathrm{s}}\|\mathbf{F} \mathbf{w}\|^{2}+\sigma_{z}^{2}\|\mathbf{w}\|^{2} .
$$

Combining Equations 10 14, the optimal forwarding matrix design problem in DRM can be transformed as

$$
\begin{aligned}
\mathcal{Q}^{\mathrm{DRM}}: & \min _{\mathbf{w}} p_{\mathrm{s}}\|\mathbf{F} \mathbf{w}\|^{2}+\sigma_{z}^{2}\|\mathbf{w}\|^{2} \\
& \mathcal{C}_{1}: \operatorname{Pr}\left\{\frac{p_{\mathrm{s}}\left|\mathbf{h}_{\mathrm{c}, k}^{H} \mathbf{F} \mathbf{w}\right|^{2}}{\sigma_{z}^{2}\left|\mathbf{h}_{\mathrm{c}, k}^{H} \mathbf{w}\right|^{2}+\sigma_{z}^{2}}<\gamma_{\mathrm{th}}\right\} \leq \rho_{\mathrm{c}}, \forall k \\
& \mathcal{C}_{2}: \operatorname{Pr}\left\{p_{\mathrm{s}}\left|\mathbf{h}_{\mathrm{p}, l}^{H} \mathbf{F w}\right|^{2}+\sigma_{z}^{2}\left|\mathbf{h}_{\mathrm{p}, l}^{H} \mathbf{w}\right|^{2}>I_{\mathrm{th}, l}\right\} \leq \rho_{\mathrm{p}}, \forall l \\
& \mathcal{C}_{3}: p_{\mathrm{s}}\|\mathbf{F} \mathbf{w}\|^{2}+\sigma_{z}^{2}\|\mathbf{w}\|^{2} \leq P_{\mathrm{th}} \\
& \mathcal{C}_{4}: \mathbf{w} \in \mathbb{C}^{N \times 1}
\end{aligned}
$$


4.2 Closed-form expressions for constraints $\mathcal{C}_{1}$ and $\mathcal{C}_{2}$ Based on the imperfect CSIT model in Equation 1 and the SINR expression in Equation 12, let $\mathbf{X}=\mathbf{w} \mathbf{w}^{H}$, and it is derived that

$$
\begin{aligned}
& \operatorname{SINR}_{k}^{\mathrm{DRM}}<\gamma_{\mathrm{th}} \\
\Leftrightarrow & \frac{p_{\mathrm{s}}}{\gamma_{\mathrm{th}}}\left|\mathbf{h}_{\mathrm{c}, k}^{H} \mathbf{F w}\right|^{2}-\sigma_{z}^{2}\left|\mathbf{h}_{\mathrm{c}, k}^{H} \mathbf{w}\right|^{2}<\sigma_{z}^{2} \\
\Leftrightarrow & \frac{p_{\mathrm{s}}}{\gamma_{\mathrm{th}}} \mathbf{h}_{\mathrm{c}, k}^{H}\left(\mathbf{F w} \mathbf{w}^{H} \mathbf{F}^{H}\right) \mathbf{h}_{\mathrm{c}, k}-\sigma_{z}^{2} \mathbf{h}_{\mathrm{c}, k}^{H} \mathbf{w} \mathbf{w}^{H} \mathbf{h}_{\mathrm{c}, k}<\sigma_{z}^{2} \\
\Leftrightarrow & \left(\hat{\mathbf{h}}_{\mathrm{c}, k}+\mathbf{e}_{\mathrm{c}, k}\right)^{H} \mathbf{A}_{\mathrm{c}}\left(\hat{\mathbf{h}}_{\mathrm{c}, k}+\mathbf{e}_{\mathrm{c}, k}\right)<\sigma_{z}^{2} \\
\Leftrightarrow & \hat{\mathbf{h}}_{\mathrm{c}, k}^{H} \mathbf{A}_{\mathrm{c}} \hat{\mathbf{h}}_{\mathrm{c}, k}+\hat{\mathbf{h}}_{\mathrm{c}, k}^{H} \mathbf{A}_{\mathrm{c}} \mathbf{e}_{\mathrm{c}, k}+\mathbf{e}_{\mathrm{c}, k}^{H} \mathbf{A}_{\mathrm{c}} \hat{\mathbf{h}}_{\mathrm{c}, k}+\mathbf{e}_{\mathrm{c}, k}^{H} \mathbf{A}_{\mathrm{c}} \mathbf{e}_{\mathrm{c}, k}<\sigma_{z}^{2} \\
\Leftrightarrow & \mathbf{e}_{\mathrm{c}, k}^{H} \mathbf{A}_{\mathrm{c}} \mathbf{e}_{\mathrm{c}, k}+2 \operatorname{Re}\left[\hat{\mathbf{h}}_{\mathrm{c}, k}^{H} \mathbf{A}_{\mathrm{c}} \mathbf{e}_{\mathrm{c}, k}\right]+\hat{\mathbf{h}}_{\mathrm{c}, k}^{H} \mathbf{A}_{\mathrm{c}} \hat{\mathbf{h}}_{\mathrm{c}, k}<\sigma_{z}^{2}
\end{aligned}
$$

where $\mathbf{A}_{\mathrm{c}}=\frac{p_{\mathrm{s}}}{\gamma_{\mathrm{th}}} \mathbf{F} \mathbf{X} \mathbf{F}^{H}-\sigma_{z}^{2} \mathbf{X}$, and the last step is obtained due to that $\mathbf{A}_{\mathrm{C}}$ is a Hermitian matrix.

Let $\tilde{\mathbf{e}}_{\mathrm{c}, k}=\frac{1}{\sigma_{\mathrm{c}, k}} \mathbf{e}_{\mathrm{c}, k}, \tilde{\mathbf{h}}_{\mathrm{c}, k}=\frac{1}{\sigma_{\mathrm{c}, k}} \hat{\mathbf{h}}_{\mathrm{c}, k}, c_{\mathrm{c}, k}=\frac{1}{\sigma_{\mathrm{c}, k}^{2}}$ $\left(\sigma_{z}^{2}-\hat{\mathbf{h}}_{\mathrm{c}, k}^{H} \mathbf{A}_{\mathrm{c}} \hat{\mathbf{h}}_{\mathrm{c}, k}\right)$, and $\mathcal{C}_{1}$ can be rewritten as

$\mathcal{C}_{1}: \operatorname{Pr}\left\{\tilde{\mathbf{e}}_{\mathrm{c}, k}^{H} \mathbf{A}_{\mathrm{c}} \tilde{\mathbf{e}}_{\mathrm{c}, k}+2 \operatorname{Re}\left[\tilde{\mathbf{h}}_{k}^{H} \mathbf{A}_{\mathrm{c}} \tilde{\mathbf{e}}_{\mathrm{c}, k}\right]<c_{\mathrm{c}, k}\right\} \leq \rho_{\mathrm{c}}, \forall k$

Using Lemma 2 in [19], $\mathcal{C}_{1}$ can be safely approximated as

$\mathcal{C}_{1}^{\prime}:\left\{\begin{array}{l}\operatorname{Tr}\left(\mathbf{A}_{\mathrm{c}}\right)-\sqrt{2 \beta_{\mathrm{c}}} x_{\mathrm{c}, k}-2 \beta_{\mathrm{c}} y_{\mathrm{c}, k} \geq c_{\mathrm{c}, k} \\ \left(\left\|\mathbf{A}_{\mathrm{c}}\right\|^{2}+2\left\|\mathbf{A}_{\mathrm{c}} \tilde{\mathbf{h}}_{\mathrm{c}, k}\right\|^{2}\right)^{1 / 2} \leq x_{\mathrm{c}, k} \quad, k=1,2, \ldots, K \\ y_{\mathrm{c}, k} \mathbf{I}+\mathbf{A}_{\mathrm{c}} \succeq \mathbf{0}\end{array}\right.$

where $\beta_{\mathrm{c}}=-\ln \rho_{\mathrm{c}}$, and $x_{\mathrm{c}, k} \geq 0, y_{\mathrm{c}, k} \geq 0$ are some new added variables. Here, $\beta_{\mathrm{c}}$ could be deemed as a penalty factor for the uncertainty of $\tilde{\mathbf{e}}_{\mathrm{c}, k}$. Note that if constraint $\mathcal{C}_{1}^{\prime}$ is satisfied, then constraint $\mathcal{C}_{1}$ is satisfied. Proof for detailed derivation could be found in Lemma 2 in [19]. In this sense, we state that the constraint transformation is safe.

Similarly, based on Equations 2 and 13, we can obtain

$$
\begin{aligned}
& I_{l}^{\mathrm{DRM}}>I_{\mathrm{th}, l} \\
\Leftrightarrow & \left(\hat{\mathbf{h}}_{\mathrm{p}, l}+\mathbf{e}_{\mathrm{p}, l}\right)^{H} \mathbf{A}_{\mathrm{p}}\left(\hat{\mathbf{h}}_{\mathrm{p}, l}+\mathbf{e}_{\mathrm{p}, l}\right)>I_{\mathrm{th}, l} \\
\Leftrightarrow & \mathbf{e}_{\mathrm{p}, l}^{H} \mathbf{A}_{\mathrm{p}} \mathbf{e}_{\mathrm{p}, l}+2 \operatorname{Re}\left[\hat{\mathbf{h}}_{\mathrm{p}, l}^{H} \mathbf{A}_{\mathrm{p}} \mathbf{e}_{\mathrm{p}, l}\right]+\hat{\mathbf{h}}_{\mathrm{p}, l}^{H} \mathbf{A}_{\mathrm{p}} \hat{\mathbf{h}}_{\mathrm{p}, l}>I_{\mathrm{th}, l}
\end{aligned}
$$

where $\mathbf{A}_{\mathrm{p}}=p_{\mathrm{s}} \mathbf{F X F ^ { H }}+\sigma_{z}^{2} \mathbf{X}$.
Let $\tilde{\mathbf{e}}_{\mathrm{p}, l}=\frac{1}{\sigma_{\mathrm{p}, l}} \mathbf{e}_{\mathrm{p}, l}, \quad \tilde{\mathbf{h}}_{\mathrm{p}, l}=\frac{1}{\sigma_{\mathrm{p}, l}} \hat{\mathbf{h}}_{\mathrm{p}, l}, \quad c_{\mathrm{p}, l}=$ $\frac{1}{\sigma_{\mathrm{p}, l}^{2}}\left(I_{\mathrm{th}, l}-\hat{\mathbf{h}}_{\mathrm{p}, l}^{H} \mathbf{A}_{\mathrm{p}} \hat{\mathbf{h}}_{\mathrm{p}, l}\right), \beta_{\mathrm{p}}=-\ln \rho_{\mathrm{p}}$. Then, constraint $\mathcal{C}_{2}$ can be rewritten as

$$
\mathcal{C}_{2}: \operatorname{Pr}\left\{\tilde{\mathbf{e}}_{\mathrm{p}, l}^{H} \mathbf{A}_{\mathrm{p}} \tilde{\mathbf{e}}_{\mathrm{p}, l}+2 \operatorname{Re}\left[\tilde{\mathbf{h}}_{\mathrm{p}, l}^{H} \mathbf{A}_{\mathrm{p}} \tilde{\mathbf{e}}_{\mathrm{p}, l}\right]>c_{\mathrm{p}, l}\right\} \leq \rho_{\mathrm{p}}, \forall l
$$

which can also be safely transformed into the following closed-form expression with the added variables $x_{\mathrm{p}, l} \geq 0$ and $y_{\mathrm{p}, l} \geq 0$.

$\mathcal{C}_{2}^{\prime}:\left\{\begin{array}{l}\operatorname{Tr}\left(\mathbf{A}_{\mathrm{p}}\right)+\sqrt{2 \beta_{\mathrm{p}}} x_{\mathrm{p}, l}+2 \beta_{\mathrm{p}} y_{\mathrm{p}, l} \leq c_{\mathrm{p}, l} \\ \left(\left\|\mathbf{A}_{\mathrm{p}}\right\|^{2}+2\left\|\mathbf{A}_{\mathrm{p}} \tilde{\mathbf{h}}_{\mathrm{p}, l}\right\|^{2}\right)^{1 / 2} \leq x_{\mathrm{p}, l} \quad, l=1,2, \ldots, L \\ y_{\mathrm{p}, l} \mathbf{I}-\mathbf{A}_{\mathrm{p}} \geq \mathbf{0}\end{array}\right.$

Furthermore, the total consumed power can be rewritten as

$$
P_{\mathrm{R}}=\operatorname{Tr}(\mathbf{B X}) \text {, where } \mathbf{B}=p_{\mathrm{s}} \mathbf{F}^{H} \mathbf{F}+\sigma_{z}^{2} \mathbf{I} \text {. }
$$

Therefore, problem $\mathcal{Q}^{\mathrm{DRM}}$ can be conservatively transformed as

$$
\begin{aligned}
\mathcal{Q}_{1}^{\mathrm{DRM}}: \quad & \min \operatorname{Tr}(\mathbf{B X}) \\
& \mathcal{C}_{1, x_{\mathrm{c}, k}, y_{\mathrm{c}, k}, \mathcal{C}_{\mathrm{p}, l}, y_{\mathrm{p}, l}}^{\prime} \\
& \mathcal{C}_{3}^{\prime}: \operatorname{Tr}(\mathbf{B X}) \leq P_{\mathrm{th}} \\
& \mathcal{C}_{4}^{\prime}: \mathbf{X} \succeq \mathbf{0}, \operatorname{Rank}(\mathbf{X})=1 \\
& \mathcal{C}_{5}^{\prime}: x_{\mathrm{c}, k} \geq 0, y_{\mathrm{c}, k} \geq 0, x_{\mathrm{p}, l} \geq 0, y_{\mathrm{p}, l} \geq 0, \forall k, l
\end{aligned}
$$

\section{Remark 2.}

- As constraints $\mathcal{C}_{1}^{\prime}$ and $\mathcal{C}_{2}^{\prime}$ are only sufficient conditions for $\mathcal{C}_{1}$ and $\mathcal{C}_{2}$, the optimal value of $\mathcal{Q}_{1}^{\mathrm{DRM}}$ is only an upper bound of the minimum power consumption of the original problem $\mathcal{Q}^{\mathrm{DRM}}$.

- Since the maximum outage probability constraint $\mathcal{C}_{1}^{\prime}$ and the maximum over-interference probability constraint $\mathcal{C}_{2}^{\prime}$ provide a lower bound and an upper bound for the power consumption $\operatorname{Tr}(\mathbf{B X})$ respectively, problem $\mathcal{Q}_{1}^{\mathrm{DRM}}$ is not always feasible.

\subsection{Detailed algorithm design}

Problem $\mathcal{Q}_{1}^{\mathrm{DRM}}$ is non-convex due to the rank-one constraint, i.e., $\operatorname{Rank}(\mathbf{X})=1$. Fortunately, some effective methods have been proposed to cope with this kind of multicast beamforming problem with rank-one constraints, including semidefinite relaxation (SDR) [17,20], second-order cone programming (SOCP) [5,21], PenFun method $[13,15]$ and so on.

As the most frequently used method, the SDR method is simple to implement and the complexity is relatively low. By comparison, the PenFun method can approximate the optimal solution better with an increased complexity. 
Therefore, in the following, we first analyze the SDR algorithm briefly as a benchmark algorithm and then present the PenFun algorithm in detail as an advanced algorithm.

\subsubsection{SDR algorithm}

In the SDR algorithm, we first relax the problem and solve the relaxed problem to obtain a relaxed optimal solution. As the relaxed solution is always close to the original optimal solution for the non-relaxed problem, we can generate feasible solutions around the relaxed solution and select the best one. With regard to the formulated problem $\mathcal{Q}_{1}^{\mathrm{DRM}}$, the following two successive steps are executed to acquire a suboptimal solution.

In the first step, the rank-one constraint is dropped, after which problem $\mathcal{Q}_{1}^{\text {DRM }}$ turns to be a convex problem and could be solved by the frequently used convex optimization methods, for example, the interior point algorithm. In this paper, the semi-definite programming (SDP) [17] is adopted to solve the relaxed problem. The detailed algorithm procedure is omitted as it is not the focus of this paper, and the optimal solution obtained from SDP is denoted as $\mathbf{X}_{\text {opt }}$.

Then, in the second step, a randomization process is implemented to obtain a series of candidate $\mathbf{w}(i), i=$ $1,2, \ldots, T$ according to $\mathbf{X}_{\text {opt }}[22]$. For the $i$ th randomization, the following sub-steps are executed.

1) Make the singular value decomposition (SVD): $\mathbf{X}_{\mathrm{opt}}=\mathbf{U S V}^{H}$ and randomly generate a normalized beamforming vector $\tilde{\mathbf{w}}(i)$ using $\mathbf{x} \sim \mathcal{C N}(\mathbf{0}, \mathbf{I})$.

$$
\hat{\mathbf{w}}(i)=\mathbf{U} \mathbf{S}^{1 / 2} \mathbf{x}, \tilde{\mathbf{w}}(i)=\frac{1}{\|\hat{\mathbf{w}}(i)\|} \hat{\mathbf{w}}(i)
$$

2) Scale $\tilde{\mathbf{w}}(i)$ with a minimum factor $\sqrt{p(i)}$ to satisfy constraints $\mathcal{C}_{1}^{\prime} \sim \mathcal{C}_{3}^{\prime}$ if there exists a feasible solution.

3) Compute the candidate beamforming vector and the power consumption as

$$
\mathbf{w}(i)=\sqrt{p(i)} \tilde{\mathbf{w}}(i), P_{\mathrm{c}}(i)=\operatorname{Tr}\left(\mathbf{B w}(i) \mathbf{w}(i)^{H}\right) .
$$

Finally, from those feasible candidates obtained in the randomization process, select the optimal $\mathbf{w}(i)$ with the minimum power consumption $P_{\mathrm{c}}(i)$ at the cognitive relay.

\subsubsection{PenFun algorithm}

The most related work concerning the PenFun method is [13], in which the authors transform the rank-one constraint into a concave penalty in the objective function and then handle the transformed problem with the convex-concave processing (CCP). However, this work only applies to perfect CSIT models, and in our paper, the algorithm is extended to the imperfect CSIT model.
Obviously, as $\mathbf{X} \geq \mathbf{0}$, the rank-one constraint can be rewritten as

$$
\operatorname{Tr}(\mathbf{X})-\lambda_{\max }(\mathbf{X}) \leq 0
$$

Furthermore, substitute this constraint by adding a penalty into the objective function. Then, problem $\mathcal{Q}_{1}^{\mathrm{DRM}}$ can be reformulated as

$$
\begin{aligned}
\mathcal{Q}_{2}^{\text {DRM }}: & \min _{\mathbf{X}, x_{\mathrm{c}, k}, y_{\mathrm{c}, k}, x_{\mathrm{p}, l}, y_{\mathrm{p}, l}} f_{o}(\mathbf{X})=\operatorname{Tr}(\mathbf{B X})+\mu\left(\operatorname{Tr}(\mathbf{X})-\lambda_{\max }(\mathbf{X})\right) \\
& \text { s.t. } \mathcal{C}_{1}^{\prime} ; \mathcal{C}_{2}^{\prime} ; \mathcal{C}_{3}^{\prime} ; \mathcal{C}_{5}^{\prime} ; \mathcal{C}_{4}^{\prime \prime}: \mathbf{X} \succeq \mathbf{0}
\end{aligned}
$$

where $\mu>0$ is a large enough weight to result in small $\operatorname{Tr}(\mathbf{X})-\lambda_{\max }(\mathbf{X})$. As $f_{o}(\mathbf{X})$ is concave, problem $\mathcal{Q}_{2}^{\mathrm{DRM}}$ is minimization of a concave function over a convex set, which can be efficiently solved with CCP $[13,15]$.

The core idea of CCP is to decompose the optimization of a non-convex problem into an iterative procedure, and in each iteration, the problem is modified as a convex one by replacing the concave part in the objective function with a Taylor's approximation. Specially, if $\mathbf{X}^{(t)}$ is the solution in the $t$ th iteration, we can approximate the objective function $f_{o}(\mathbf{X})$ in the $(t+1)$ th iteration as follows.

$$
\begin{aligned}
f_{a}(\mathbf{X})= & \operatorname{Tr}(\mathbf{B X})+\mu\{\operatorname{Tr}(\mathbf{X}) \\
& \left.-\lambda_{\max }\left(\mathbf{X}^{(t)}\right)-\operatorname{Tr}\left[\partial \mathbf{X}^{(t)}\left(\mathbf{X}-\mathbf{X}^{(t)}\right)\right]\right\}
\end{aligned}
$$

with $\partial \mathbf{X}^{(t)}$ denoting the sub-gradient of $\lambda_{\max }\left(\mathbf{X}^{(t)}\right)$, which can be selected as

$$
\partial \mathbf{X}^{(t)}=\mathbf{x}_{\max } \mathbf{x}_{\max }^{H},
$$

where $\mathbf{x}_{\max }$ is the normalized principal eigenvector of $\mathbf{X}^{(t)}$, i.e., the eigenvector corresponding to the maximal eigenvalue $\lambda_{\max }\left(\mathbf{X}^{(t)}\right)$.

Therefore, the optimization problem in the $(t+1)$ th iteration can be expressed as

$$
\begin{array}{ll}
\mathcal{Q}_{3}^{\mathrm{DRM}}: & \min {\mathrm{x}, x_{\mathrm{c}, k}, y_{\mathrm{c}, k}, x_{\mathrm{p}, l}, y_{\mathrm{p}, l}}_{a}(\mathbf{X}) \\
& \text { s.t. } \mathcal{C}_{1}^{\prime} ; \mathcal{C}_{2}^{\prime} ; \mathcal{C}_{3}^{\prime} ; \mathcal{C}_{4}^{\prime \prime} ; \mathcal{C}_{5}^{\prime}
\end{array}
$$

Problem $\mathcal{Q}_{3}^{\text {DRM }}$ is convex and can be efficiently solved by the SDP method [17]. The whole process of the PenFun algorithm for problem $\mathcal{Q}_{1}^{\mathrm{DRM}}$ is summarized as follows.

\section{Input:}

1: $\mu, t=0, \mathbf{X}^{(0)}, \varepsilon_{d}, \varepsilon_{\Delta}$.

Initialization:

2: Calculate $d=\operatorname{Tr}\left(\mathbf{X}^{(t)}\right)-\lambda_{\max }\left(\mathbf{X}^{(t)}\right)$. If $d \leq \varepsilon_{d}$, terminate the initialization stage. 
3: Calculate $\lambda_{\max }\left(\mathbf{X}^{(t)}\right), \mathbf{x}_{\max }$ and solve problem $\mathcal{Q}_{3}^{\mathrm{DRM}}$, acquiring the optimal $\mathbf{X}^{(t+1)}$.

4: Calculate $\Delta=\left\|\mathbf{X}^{(t+1)}-\mathbf{X}^{(t)}\right\|$. If $\Delta \leq \varepsilon_{\Delta}$, let $\mu=2 \mu$. 5: Set $t=t+1$, and go back to step 2 .

\section{Iteration:}

6: Calculate $\lambda_{\max }\left(\mathbf{X}^{(t)}\right), \mathbf{x}_{\max }$ and solve problem $\mathcal{Q}_{3}^{\mathrm{DRM}}$, acquiring the optimal solution $\mathbf{X}^{(t+1)}$.

7: Calculate $\Delta=\left\|\mathbf{X}^{(t+1)}-\mathbf{X}^{(t)}\right\|$. If $\Delta \leq \varepsilon_{\Delta}$, terminate the Iteration stage.

8: Set $t=t+1$, and go back to step 6 .

\section{Algorithm design for CRM}

In this section, Lemma 1 is presented to show that the optimal $\mathbf{W}$ in CRM is a rank-one matrix. Specially, $\mathbf{W}$ is the combination of the matched-filter receiver $\mathbf{f}^{H}$ and the adaptive beamformer $\mathbf{w}$. Based on this lemma, the matrix optimization is transformed into the beamformer design, which is similar to the problem in DRM. Finally, the Bernstein-type inequality and the PenFun method are jointly employed to solve the beamformer design problem.

\subsection{Optimal structure of the forwarding matrix $\mathbf{W}$}

Lemma 1. The optimal forwarding matrix $\mathbf{W}$ has the following structure.

$$
\mathbf{W}=\mathbf{w f}^{H}
$$

\section{Proof. See Appendix A.}

Lemma 1 indicates that the optimal forwarding matrix $\mathbf{W}$ is the combination of a matched-filter receiver $\mathbf{f}^{H}$ and an adaptive beamformer $\mathbf{w}$ as shown in Figure 2. In other words, we first receive the signal with maximal ratio combining (MRC) to achieve the best signal quality and then precode the received signal with a suitable beamforming vector to balance the received power at different cognitive destinations.
Based on the optimal structure, Equations $6 \sim 8$ can be rewritten as

$$
\begin{aligned}
& \operatorname{SINR}_{k}^{C R M}=\frac{p_{\mathrm{s}}\|\mathbf{f}\|^{4}\left|\mathbf{h}_{\mathrm{c}, k}^{H} \mathbf{w}\right|^{2}}{\sigma_{z}^{2}\|\mathbf{f}\|^{2}\left|\mathbf{h}_{\mathrm{c}, k}^{H} \mathbf{w}\right|^{2}+\sigma_{z}^{2}}, \\
& I_{l}^{\mathrm{CRM}}=\left(p_{\mathrm{s}}\|\mathbf{f}\|^{4}+\sigma_{z}^{2}\|\mathbf{f}\|^{2}\right)\left|\mathbf{h}_{\mathrm{p}, l}^{H} \mathbf{w}\right|^{2},
\end{aligned}
$$

$$
P_{\mathrm{R}}=\left(p_{\mathrm{s}}\|\mathbf{f}\|^{4}+\sigma_{z}^{2}\|\mathbf{f}\|^{2}\right)\|\mathbf{w}\|^{2}
$$

Let $\tilde{\gamma}_{\text {th }}=\frac{\gamma_{\text {th }} \sigma_{z}^{2}}{p_{\mathrm{s}}\|\mathbf{f}\|^{4}-\gamma_{\text {th }} \sigma_{z}^{2}\|\mathbf{f}\|^{2}}$, it is derived that

$$
\operatorname{SINR}_{k}^{\mathrm{CRM}}<\gamma_{\text {th }} \Leftrightarrow\left|\mathbf{h}_{\mathrm{c}, k}^{H} \mathbf{w}\right|^{2}<\tilde{\gamma}_{\text {th }}
$$

Moreover, define $\alpha=p_{s}\|\mathbf{f}\|^{4}+\sigma_{z}^{2}\|\mathbf{f}\|^{2}, \tilde{I}_{\mathrm{th}, l}=I_{\mathrm{th}, l} / \alpha$, problem $\mathcal{Q}^{\text {CRM }}$ can be rewritten as follows.

$$
\begin{aligned}
\mathcal{Q}^{\mathrm{CRM}}: \quad & \min _{\mathbf{w}} \alpha\|\mathbf{w}\|^{2} \\
& \mathcal{C}_{1}: \operatorname{Pr}\left\{\left|\mathbf{h}_{\mathrm{c}, k}^{H} \mathbf{w}\right|^{2}<\tilde{\gamma}_{\mathrm{th}}\right\} \leq \rho_{\mathrm{c}}, \forall k \\
& \mathcal{C}_{2}: \operatorname{Pr}\left\{\left|\mathbf{h}_{\mathrm{p}, l}^{H} \mathbf{w}\right|^{2}>\tilde{I}_{\mathrm{th}, l}\right\} \leq \rho_{\mathrm{p}}, \forall l \\
& \mathcal{C}_{3}: \alpha\|\mathbf{w}\|^{2} \leq P_{\mathrm{th}} \\
& \mathcal{C}_{3}: \mathbf{w} \in \mathbb{C}^{N \times 1}
\end{aligned}
$$

Obviously, $\tilde{\gamma}_{\text {th }} \geq 0$ is required in practice, and hence the maximum achieved transmission SINR $\gamma_{\text {th }}$ is $p_{\mathrm{s}}\|\mathbf{f}\|^{2} / \sigma_{z}^{2}$, i.e.,

$$
\gamma_{\text {th }} \leq \gamma_{\max }=p_{\mathrm{s}}\|\mathbf{f}\|^{2} / \sigma_{z}^{2}
$$

This is because the achieved transmission SINR is limited by the channel gain from the cognitive source to the cognitive relay.

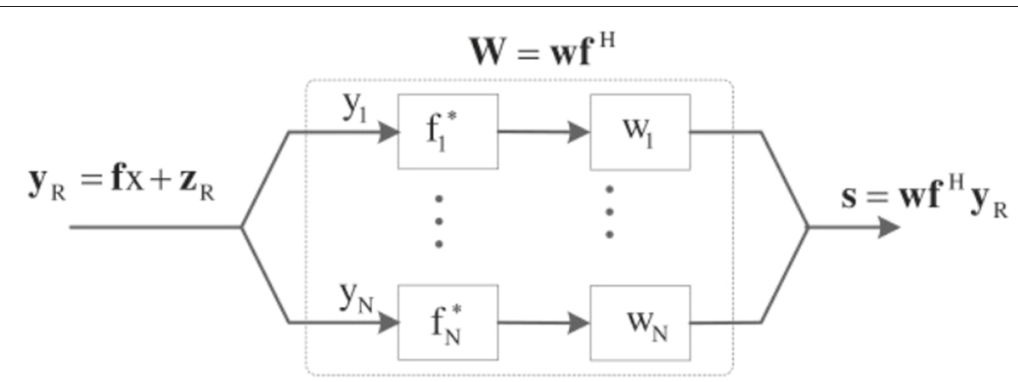

Figure 2 Optimal structure of the forwarding matrix at the relay node. The optimal forwarding matrix in CRM is the combination of the match-filter receiver and the adaptive beamformer. In other words, the cognitive relay first maximizes the received signal quality with maximal ratio combining and then precodes the received signal with a beamforming vector $\mathbf{w}$ to balance the received power at different cognitive destinations and reduce the received interference at primary users. 
5.2 Closed-form expressions for constraints $\mathcal{C}_{1}$ and $\mathcal{C}_{2}$ Similar to the analysis for DRM, let $\mathbf{X}=\mathbf{w w}^{H}$, and the following expression can be derived based on the imperfect CSIT model in Equation 1.

$$
\begin{aligned}
\left|\mathbf{h}_{\mathrm{c}, k}^{H} \mathbf{w}\right|^{2} & =\left(\hat{\mathbf{h}}_{\mathrm{c}, k}+\mathbf{e}_{\mathrm{c}, k}\right)^{H} \mathbf{w} \mathbf{w}^{H}\left(\hat{\mathbf{h}}_{\mathrm{c}, k}+\mathbf{e}_{\mathrm{c}, k}\right) \\
& =\mathbf{e}_{\mathrm{c}, k}^{H} \mathbf{X} \mathbf{e}_{\mathrm{c}, k}+2 \operatorname{Re}\left[\hat{\mathbf{h}}_{\mathrm{c}, k}^{H} \mathbf{X} \mathbf{e}_{\mathrm{c}, k}\right]+\hat{\mathbf{h}}_{\mathrm{c}, k}^{H} \mathbf{X} \hat{\mathbf{h}}_{\mathrm{c}, k} .
\end{aligned}
$$

Let $\tilde{\mathbf{e}}_{\mathrm{c}, k}=\frac{1}{\sigma_{\mathrm{c}, k}} \mathbf{e}_{\mathrm{c}, k}, \quad \tilde{\mathbf{h}}_{\mathrm{c}, k}=\frac{1}{\sigma_{\mathrm{c}, k}} \hat{\mathbf{h}}_{\mathrm{c}, k}, \quad c_{\mathrm{c}, k}=$ $\frac{1}{\sigma_{\mathrm{c}, k}^{2}}\left(\tilde{\gamma}_{\mathrm{th}}-\hat{\mathbf{h}}_{\mathrm{c}, k}^{H} \mathbf{X h}_{\mathrm{c}, k}\right), \mathcal{C}_{1}$ can be rewritten as

$$
\mathcal{C}_{1}: \operatorname{Pr}\left\{\tilde{\mathbf{e}}_{\mathrm{c}, k}^{H} \mathbf{X} \tilde{\mathbf{e}}_{\mathrm{c}, k}+2 \operatorname{Re}\left[\tilde{\mathbf{h}}_{\mathrm{c}, k}^{H} \mathbf{X} \tilde{\mathbf{e}}_{\mathrm{c}, k}\right]<c_{\mathrm{c}, k}\right\} \leq \rho_{\mathrm{c}}, \forall k
$$

Using Lemma 2 in [19], $\mathcal{C}_{1}$ can be safely transformed into

$\mathcal{C}_{1}^{\prime}:\left\{\begin{array}{l}\operatorname{Tr}(\mathbf{X})-\sqrt{2 \beta_{\mathrm{c}}} x_{\mathrm{c}, k}-2 \beta_{\mathrm{c}} y_{\mathrm{c}, k} \geq c_{\mathrm{c}, k} \\ \left(\|\mathbf{X}\|^{2}+2\left\|\mathbf{X} \tilde{\mathbf{h}}_{\mathrm{c}, k}\right\|^{2}\right)^{1 / 2} \leq x_{\mathrm{c}, k} \quad, k=1,2, \ldots, K \\ y_{\mathrm{c}, k} \mathbf{I}+\mathbf{X} \geq \mathbf{0}\end{array} \quad\right.$,

where $\beta_{\mathrm{c}}=-\ln \rho_{\mathrm{c}}$, and $x_{\mathrm{c}, k} \geq 0, y_{\mathrm{c}, k} \geq 0$ are some new added variables.

Moreover,

$$
\begin{aligned}
\left|\mathbf{h}_{\mathrm{p}, l}^{H} \mathbf{w}\right|^{2} & =\left(\hat{\mathbf{h}}_{\mathrm{p}, l}+\mathbf{e}_{\mathrm{p}, l}\right)^{H} \mathbf{w} \mathbf{w}^{H}\left(\hat{\mathbf{h}}_{\mathrm{p}, l}+\mathbf{e}_{\mathrm{p}, l}\right) \\
& =\mathbf{e}_{\mathrm{p}, l}^{H} \mathbf{X} \mathbf{e}_{\mathrm{p}, l}+2 \operatorname{Re}\left[\hat{\mathbf{h}}_{\mathrm{p}, l}^{H} \mathbf{X} \mathbf{e}_{\mathrm{p}, l}\right]+\hat{\mathbf{h}}_{\mathrm{p}, l}^{H} \mathbf{X} \hat{\mathbf{h}}_{\mathrm{p}, l} .
\end{aligned}
$$

Let $\tilde{\mathbf{e}}_{\mathrm{p}, l}=\frac{1}{\sigma_{\mathrm{p}, l}} \mathbf{e}_{\mathrm{p}, l}, \quad \tilde{\mathbf{h}}_{\mathrm{p}, l}=\frac{1}{\sigma_{\mathrm{p}, l}} \hat{\mathbf{h}}_{\mathrm{p}, l}, \quad c_{\mathrm{p}, l}=$ $\frac{1}{\sigma_{\mathrm{p}, l}^{2}}\left(\tilde{I}_{\mathrm{th}, l}-\hat{\mathbf{h}}_{\mathrm{p}, l}^{H} \mathbf{X} \hat{\mathbf{h}}_{\mathrm{p}, l}\right), \beta_{\mathrm{p}}=-\ln \rho_{\mathrm{p}}$. Then, constraint $\mathcal{C}_{2}$ can be rewritten as

$$
\mathcal{C}_{2}: \operatorname{Pr}\left\{\tilde{\mathbf{e}}_{\mathrm{p}, l}^{H} \mathbf{X} \tilde{\mathbf{e}}_{\mathrm{p}, l}+2 \operatorname{Re}\left[\tilde{\mathbf{h}}_{\mathrm{p}, l}^{H} \mathbf{X} \tilde{\mathbf{e}}_{\mathrm{p}, l}\right]>c_{\mathrm{p}, l}\right\} \leq \rho_{\mathrm{p}}, \forall l,
$$

which can also be safely transformed into the following closed-form expression with the added variables $x_{\mathrm{p}, l} \geq 0$ and $y_{\mathrm{p}, l} \geq 0$.

$$
\mathcal{C}_{2}^{\prime}:\left\{\begin{array}{l}
\operatorname{Tr}(\mathbf{X})+\sqrt{2 \beta_{\mathrm{p}}} x_{\mathrm{p}, l}+2 \beta_{\mathrm{p}} y_{\mathrm{p}, l} \leq c_{\mathrm{p}, l} \\
\left(\|\mathbf{X}\|^{2}+2\left\|\mathbf{X} \tilde{\mathbf{h}}_{\mathrm{p}, l}\right\|^{2}\right)^{1 / 2} \leq x_{\mathrm{p}, l} \\
y_{\mathrm{p}, l} \mathbf{I}-\mathbf{X} \geq \mathbf{0}
\end{array}\right.
$$

Combining Equations 35 and 38, problem $\mathcal{Q}^{\text {CRM }}$ can be conservatively transformed as

$$
\begin{aligned}
& \mathcal{Q}_{1}^{\mathrm{CRM}}: \quad \min _{\mathbf{X}, x_{\mathrm{c}, k}, y_{\mathrm{c}, k}, x_{\mathrm{p}, l}, y_{\mathrm{p}, l}} \alpha \operatorname{Tr}(\mathbf{X}) \\
& \mathcal{C}_{1}^{\prime}, \mathcal{C}_{2}^{\prime} \\
& \mathcal{C}_{3}^{\prime}: \alpha \operatorname{Tr}(\mathbf{X}) \leq P_{\text {th }} \\
& \mathcal{C}_{4}^{\prime}: \mathbf{X} \succeq \mathbf{0}, \operatorname{Rank}(\mathbf{X})=1 \\
& \mathcal{C}_{5}^{\prime}: x_{\mathrm{c}, k} \geq 0, y_{\mathrm{c}, k} \geq 0, x_{\mathrm{p}, l} \geq 0, y_{\mathrm{p}, l} \geq 0
\end{aligned}
$$

Similar to the analysis in Remark $2, \mathcal{Q}_{1}^{\mathrm{CRM}}$ only gives an upper bound of the original problem $\mathcal{Q}^{\mathrm{CRM}}$ as constraints $\mathcal{C}_{1}^{\prime}$ and $\mathcal{C}_{2}^{\prime}$ are sufficient but not necessary conditions for $\mathcal{C}_{1}$ and $\mathcal{C}_{2}$. Meanwhile, problem $\mathcal{Q}_{1}^{\mathrm{CRM}}$ is also not always feasible.

\subsection{Detailed algorithm design}

Similarly, both SDR and PenFun methods can be employed to solve problem $\mathcal{Q}_{1}^{\mathrm{CRM}}$. For brevity, the detailed explanation for the SDR algorithm is omitted, and in the following, we explain the PenFun algorithm simply.

First, redefine the constraint $\operatorname{Rank}(\mathbf{X})=1$ as $\operatorname{Tr}(\mathbf{X})-$ $\lambda_{\max }(\mathbf{X}) \leq 0$ and add the penalty for this constraint into the objective function.

$$
\begin{aligned}
& \mathcal{Q}_{2}^{\mathrm{CRM}}: \min _{\mathbf{X}, x_{\mathrm{c}, k}, y_{\mathrm{c}, k}, x_{\mathrm{p}, l}, y_{\mathrm{p}, l}} f_{o}(\mathbf{X})=\alpha \operatorname{Tr}(\mathbf{X})+\mu\left(\operatorname{Tr}(\mathbf{X})-\lambda_{\max }(\mathbf{X})\right) \\
& \text { s.t. } \mathcal{C}_{1}^{\prime} ; \mathcal{C}_{2}^{\prime} ; \mathcal{C}_{3}^{\prime} ; \mathcal{C}_{5}^{\prime} ; \mathcal{C}_{4}^{\prime \prime}: \mathbf{X} \succeq \mathbf{0}
\end{aligned}
$$

Then, the CCP method is adopted to solve the non-convex optimization problem $\mathcal{Q}_{2}^{\mathrm{CRM}}$, in which the optimization of problem $\mathcal{Q}_{2}^{\mathrm{CRM}}$ is decomposed into the iterative optimization of a series of convex problems.

Given that $\mathbf{X}^{(t)}$ is the solution of the convex problem in the $t$ th iteration, the objective function $f_{o}(\mathbf{X})$ in the $(t+$ 1)th iteration can be approximated as

$$
\begin{aligned}
f_{a}(\mathbf{X})= & \alpha \operatorname{Tr}(\mathbf{X})+\mu\left\{\operatorname{Tr}(\mathbf{X})-\lambda_{\max }\left(\mathbf{X}^{(t)}\right)\right. \\
& \left.-\operatorname{Tr}\left[\partial \mathbf{X}^{(t)}\left(\mathbf{X}-\mathbf{X}^{(t)}\right)\right]\right\},
\end{aligned}
$$

with $\partial \mathbf{X}^{(t)}$ denoting the sub-gradient of $\lambda_{\max }\left(\mathbf{X}^{(t)}\right)$ defined in Equation 30. And the optimization problem in the $(t+$ 1)th iteration can be expressed as

$$
\begin{array}{ll}
\mathcal{Q}_{3}^{\mathrm{CRM}}: & \min \quad f_{,, x_{\mathrm{c}, k}, y_{\mathrm{C}, k}, x_{\mathrm{p}, l}, y_{\mathrm{p}, l}} f_{a}(\mathbf{X}) \\
& \text { s.t. } \mathcal{C}_{1}^{\prime} ; \mathcal{C}_{2}^{\prime} ; \mathcal{C}_{3}^{\prime} ; \mathcal{C}_{4}^{\prime \prime} ; \mathcal{C}_{5}^{\prime}
\end{array}
$$

Problem $\mathcal{Q}_{3}^{\text {CRM }}$ can be solved with the SDP method. The detailed process of the PenFun algorithm for $\mathcal{Q}_{1}^{\mathrm{CRM}}$ is similar to the one for $\mathcal{Q}_{1}^{\mathrm{DRM}}$ and is omitted due to space limitation. 


\section{Performance evaluation}

In this paper, the channel gain between any antenna pair is modeled as $h=d^{-c / 2} e^{j \theta}$ [12], where $d$ is transmission distance, $c$, chosen as 3.5 , is the path loss exponent, and $\theta$ is uniformly distributed in $[0,2 \pi)$. The distance between the cognitive source and the cognitive relay is set as 1 , and the distance between the cognitive relay and cognitive destinations ranges from 1 to 2 uniformly. Meanwhile, for brevity, the distance from the cognitive relay to primary users is fixed as 3.

The CSIT errors for the Rayleigh channel are $\sigma_{\mathrm{c}, k}=0.1$ and $\sigma_{\mathrm{p}, l}=0.2$ by default. While calculating the overall CSIT errors, the path loss $d^{-c / 2}$ should be multiplied. The prescribed maximum outage probability threshold and the maximum over-interference probability threshold are set as $\rho_{\mathrm{c}}=\rho_{\mathrm{p}}=0.1$.

Other simulation parameters which are fixed throughout the following simulations include: $N=4, K=8$, $L=2, p_{\mathrm{s}}=1 \mathrm{~W}, \sigma_{z}^{2}=-30 \mathrm{dBW}$.

The results are averaged by 2,000 channel realizations; and for the SDR algorithm, 5,000 times of randomization are performed in each channel realization. The computer used in the simulations is Lenovo PC with the following configuration.

- Processor: Intel(R) Core(TM) i3-2130 CPU@ $3.40 \mathrm{GHz}$;

- RAM: $2 \mathrm{GHz}$ (1.8 GHz available);

- System type: a 32-bit operation system.

In Figures 3, 4, 5, 6, and 7, performance comparison with different minimum required transmission SINR is demonstrated. Four schemes are simulated, including 'DRM+SDR', 'DRM+PenFun', 'CRM+SDR', and 'CRM+PenFun'. Figures 3 and 4 plot the successful transmission probabilities and the total power consumption of different schemes. The successful transmission probability is defined as the probability that the formulated problem for DRM (CRM), i.e., $\mathcal{Q}_{1}^{\mathrm{DRM}}\left(\mathcal{Q}_{1}^{\mathrm{CRM}}\right)$, is successfully solved with the proposed SDR (PenFun) algorithm.

It is shown that no matter in DRM or CRM, the successful transmission probability of the PenFun algorithm is much higher than that of the SDR algorithm. Meanwhile, for the SDR algorithm, CRM can sustain a higher successful transmission probability than DRM. It is also found that along with the increase of the required transmission SINR $\gamma_{\text {th }}$, the average consumed power of all four schemes increases correspondingly. Moreover, CRM with the PenFun algorithm consumes the least transmit power, and DRM with the SDR method consumes the most transmit power. In addition, compared to DRM with the SDR algorithm, improving the optimization algorithm (employing the PenFun algorithm) is able to reduce more power consumption than improving the relay mode (employing CRM). In other words, during the observed range of $\gamma_{\text {th }}$, the performance gain obtained from the algorithm improvement is larger than that from the mode improvement.

Figures 5 and 6 are the comparison of the average outage probability of cognitive destinations and the average over-interference probability of primary users. To be fair, only outage probability and over-interference probability in the channel realization with successful transmission are averaged in simulations.

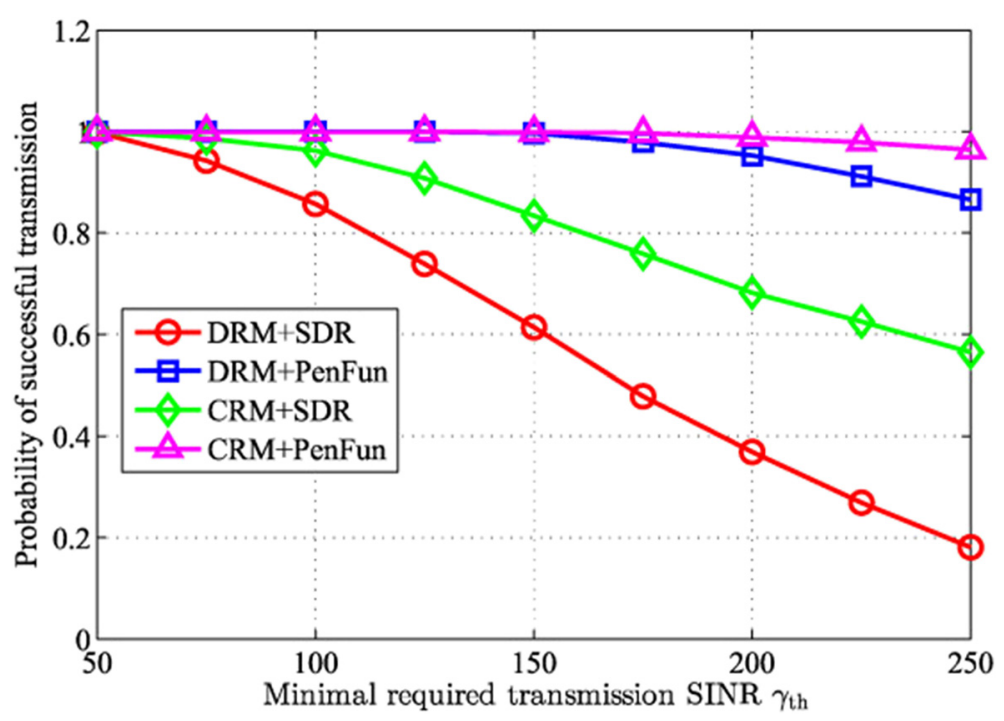

Figure 3 Probability of successful transmission with different required transmission SINR. The interference threshold is set as Ith,l=0.2 W, and the simulation results are averaged by 2,000 channel realizations. 


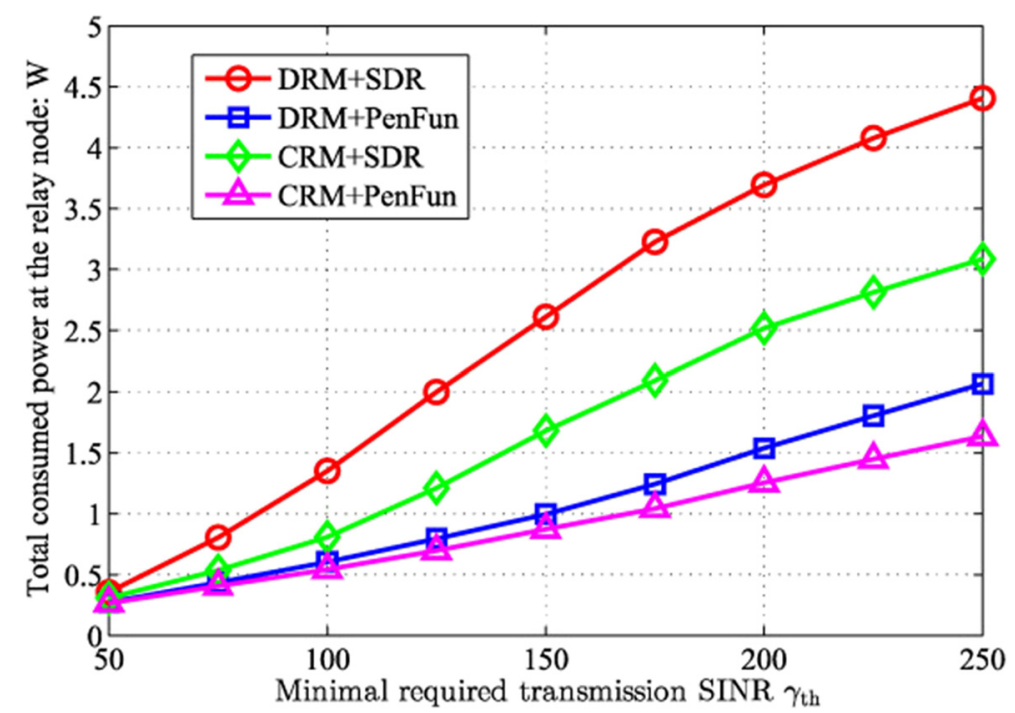

Figure 4 Total consumed power at the cognitive relay with different required transmission SINR. The interference threshold is set as $I_{\text {th }, l}=0.2 \mathrm{~W}$, and the simulation results are averaged by 2,000 channel realizations.

It is observed that when the formulated problem is feasible, all these four schemes can provide robust data reception for destinations. The maximum outage probability is about 0.006, much lower than the prescribed threshold $\rho_{\mathrm{c}}=0.1$. However, it is easy to prove that the maximum outage probability constraint $\mathcal{C}_{1}$ in problem $\mathcal{Q}$ should be satisfied tightly, indicating an achieved outage probability equal to $\rho_{\mathrm{c}}$. The achieved results do not agree with our analysis. This is because that constraint $\mathcal{C}_{1}$ is conservatively transformed into $\mathcal{C}_{1}^{\prime}$ in problems $\mathcal{Q}_{1}^{\mathrm{DRM}}$ and $\mathcal{Q}_{1}^{\mathrm{CRM}}$, and hence the achieved outage probabilities in these two transformed problems become much lower than the prescribed thresholds.

Similarly, all these schemes support perfect interference control for primary users, with the maximum overinterference probability less than 0.015. The achieved

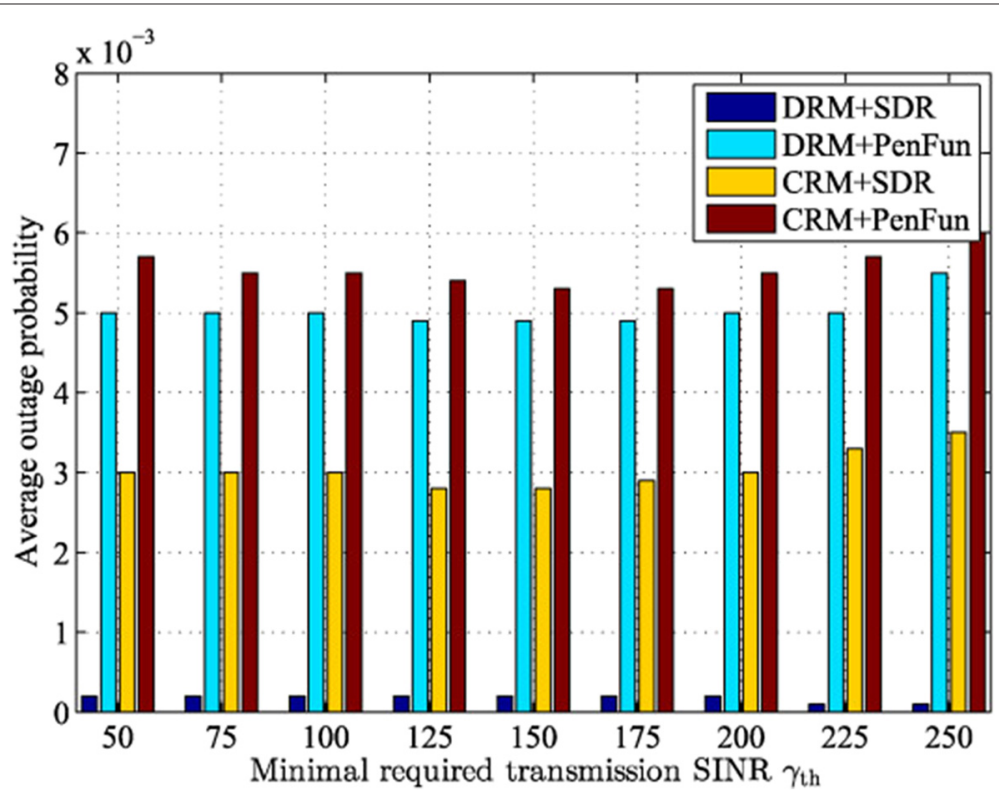

Figure 5 Average outage probability of cognitive destinations with different required transmission SINR. The interference threshold is set as $I_{\text {th }, l}=0.2 \mathrm{~W}$, and the simulation results are averaged by 2,000 channel realizations. 


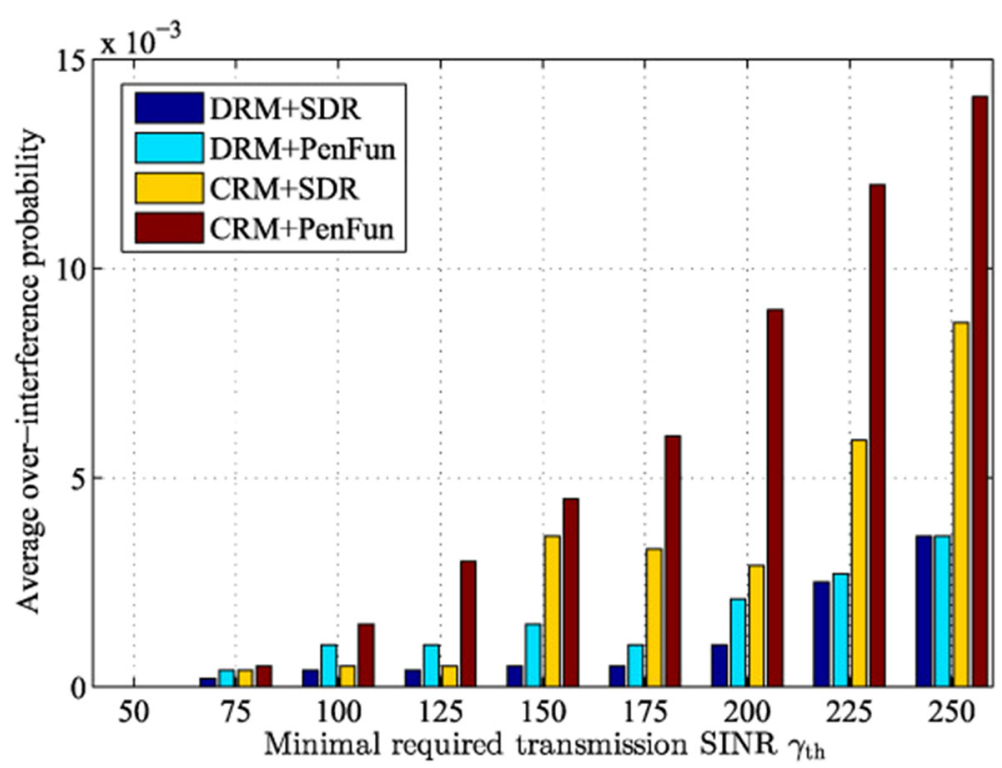

Figure 6 Average over-interference probability of cognitive destinations with different required transmission SINR. The interference threshold is set as $I_{\text {th }, l}=0.2 \mathrm{~W}$, and the simulation results are averaged by 2,000 channel realizations.

over-interference probability is much lower than the prescribed threshold $\rho_{\mathrm{p}}=0.1$ because the maximum overinterference probability constraint $\mathcal{C}_{2}$ is strengthened in the transformed problems $\mathcal{Q}_{1}^{\mathrm{DRM}}$ and $\mathcal{Q}_{1}^{\mathrm{CRM}}$.

Figure 7 shows the average computation time of different schemes. We can observe that the average computation time of the PenFun algorithm is about 3 (7.5) times higher than that of the SDR algorithm for DRM (CRM).
And moreover, DRM consumes more computation time than CRM does.

In summary, we conclude that in the observed range of $\gamma_{\text {th }}$,

1) As the optimization degree of freedom of CRM is larger than that of DRM, CRM provides better resource utilization, resulting in higher successful transmission probability and less consumed power.

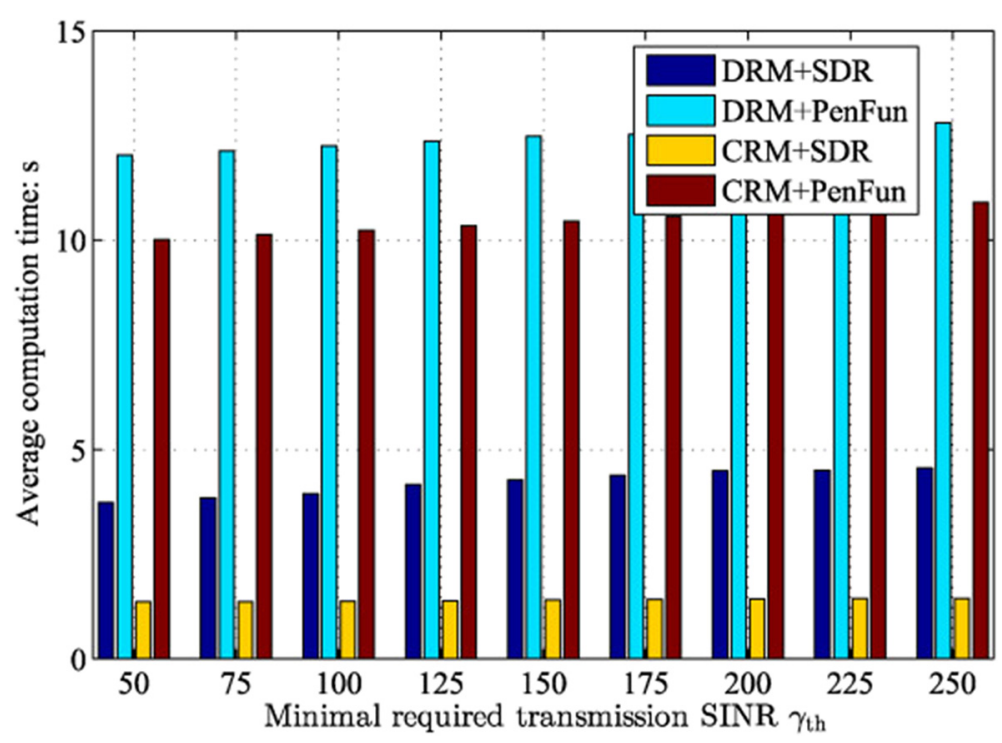

Figure 7 Average computation time with different required transmission SINR. The interference threshold is set as / th,l $=0.2 \mathrm{~W}$, and the simulation results are averaged by 2,000 channel realizations. 
2) No matter in DRM or CRM, the PenFun algorithm can achieve a much better solution than the SDR algorithm, i.e., the PenFun algorithm supports higher successful transmission probability and consumes less transmit power. However, an iterative optimization is required as shown in Sections 3.3.2 and 4.3, leading to a much higher computation complexity.

3) With respect to the successful transmission probability and the total consumed power, improving the optimization algorithm results in larger performance gain than improving the relay mode.

4) The achieved outage probability and overinterference probability in the proposed schemes are much lower than the prescribed thresholds. To approximate the original constraints and reduce the power consumption, $\beta_{\mathrm{c}}=-\ln \rho_{\mathrm{c}} / \epsilon_{\mathrm{c}}\left(\beta_{\mathrm{p}}=-\ln \rho_{\mathrm{p}} / \epsilon_{\mathrm{p}}\right)$ can be adopted in the constraint transformation, where $\epsilon_{\mathrm{c}}>1\left(\epsilon_{\mathrm{p}}>1\right)$ is the adjustment parameter, and can be obtained by the training process and the bisection search [16].

Moreover, the performance of relaying schemes which do not consider CSIT error is also simulated. In the following figures, the proposed relaying schemes are denoted as 'DRM w.c. CSIT error' and 'CRM w.c. CSIT error', where the PenFun algorithm is adopted in the simulations. By contrast, 'DRM w/o.c. CSIT error' and 'CRM w/o.c. CSIT error' denote the relaying schemes which do not consider CSIT error, and the adopted algorithms are mainly modified from [5,7] respectively.

In Figures 8, 9, 10, and 11, $\gamma_{\text {th }}=150$ is set, and the performance comparison is plotted with different interference threshold $I_{\text {th }, l}$. It is observed that although 'DRM w/o.c. CSIT error' and 'CRM w/o.c. CSIT error' acquire higher successful transmission probabilities and lower power consumption, the achieved average outage probability and over-interference probability violate the prescribed thresholds seriously. For example, when $I_{\text {th }, l}=0.03 \mathrm{~W}$, the average outage probability and over-interference probability are $0.1994(0.1952)$ and 0.3198 (0.3065) in 'DRM w/o.c. CSIT error' ('CRM w/o.c. CSIT error'), much higher than the prescribed maximum threshold $\rho_{\mathrm{c}}=\rho_{\mathrm{p}}=0.1$. Moreover, it is shown in Figure 11 that as the interference threshold decreases, the achieved over-interference probabilities in these two schemes increase greatly.

In contrast, the proposed schemes can guarantee the maximum outage probability constraint and the maximum over-interference probability constraint perfectly no matter what value $I_{\mathrm{th}, l}$ is set. The guarantee for the maximum outage probability and the maximum overinterference probability is achieved at the cost of lower successful transmission probability and larger power consumption.

\section{Conclusion}

This paper investigates the optimal forwarding strategy design for multicast transmission in cognitive relay systems with both DRM and CRM. Meanwhile, to improve the practicability of our research, imperfect CSIT feedback is considered. For DRM, we employ the Bernstein-type inequality to transform the probabilistic constraints into closed-form expressions and then utilize the SDR/PenFun method to optimize the diagonal vector.

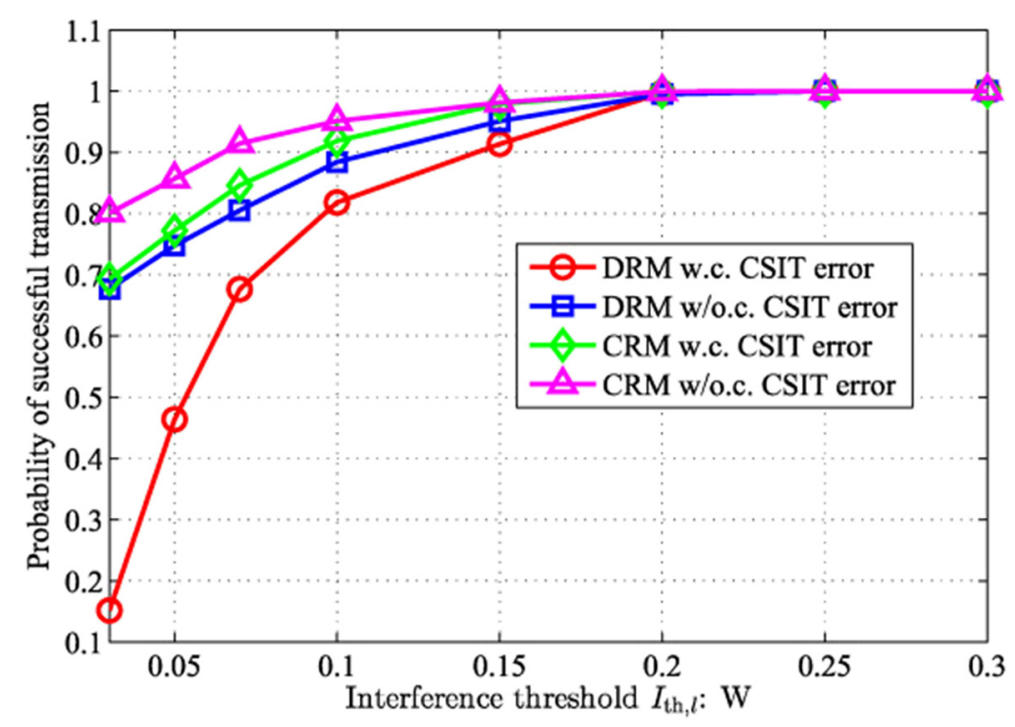

Figure 8 Probability of successful transmission with different interference thresholds. The minimum required transmission SINR is set as $\gamma_{\text {th }}=150$, and the simulation results are averaged by 2,000 channel realizations. 


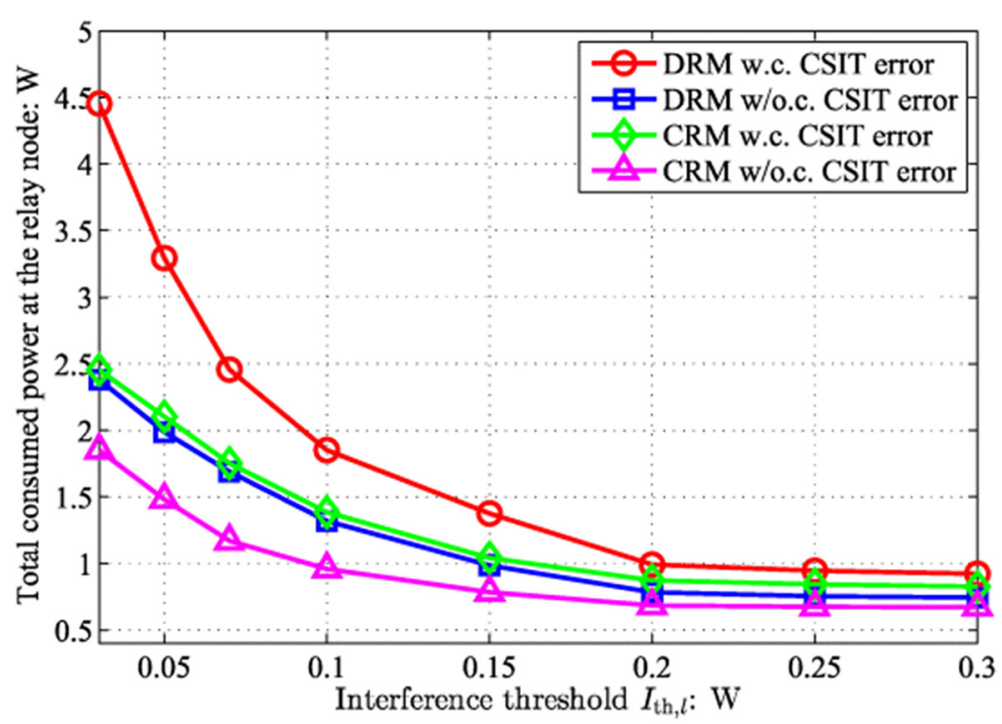

Figure 9 Total consumed power at the cognitive relay with different interference thresholds. The minimum required transmission SINR is set as $\gamma_{\text {th }}=150$, and the simulation results are averaged by 2,000 channel realizations.

For CRM, we first derive the optimal structure of the forwarding matrix and then jointly exploit the Bernsteintype inequality and the PenFun method to solve the transformed problem. Simulation results show that CRM provides higher resource utilization, and the PenFun algorithm can achieve better solution than the SDR algorithm at the cost of increased complexity. Moreover, compared with the existing schemes, which concentrate on perfect CSIT feedback model, the proposed scheme can provide a superior user experience for secondary users and protect the communications of primary users better by keeping both the outage probability for secondary users and the over-interference probability for primary users below the prescribed thresholds. However, only one multicast session and one relay node are considered in this paper. In our future work, the research will be extended to multiple mulicast sessions and multiple relay nodes.

\section{Appendix A: Proof of Lemma 1}

Let $\mathbf{H}=\left[\mathbf{h}_{\mathrm{c}, 1}, \ldots, \mathbf{h}_{\mathrm{c}, K}, \mathbf{h}_{\mathrm{p}, 1}, \ldots, \mathbf{h}_{\mathrm{p}, L}\right]$, and the optimal structure of $\mathbf{W}$ is analyzed in the following two different cases.

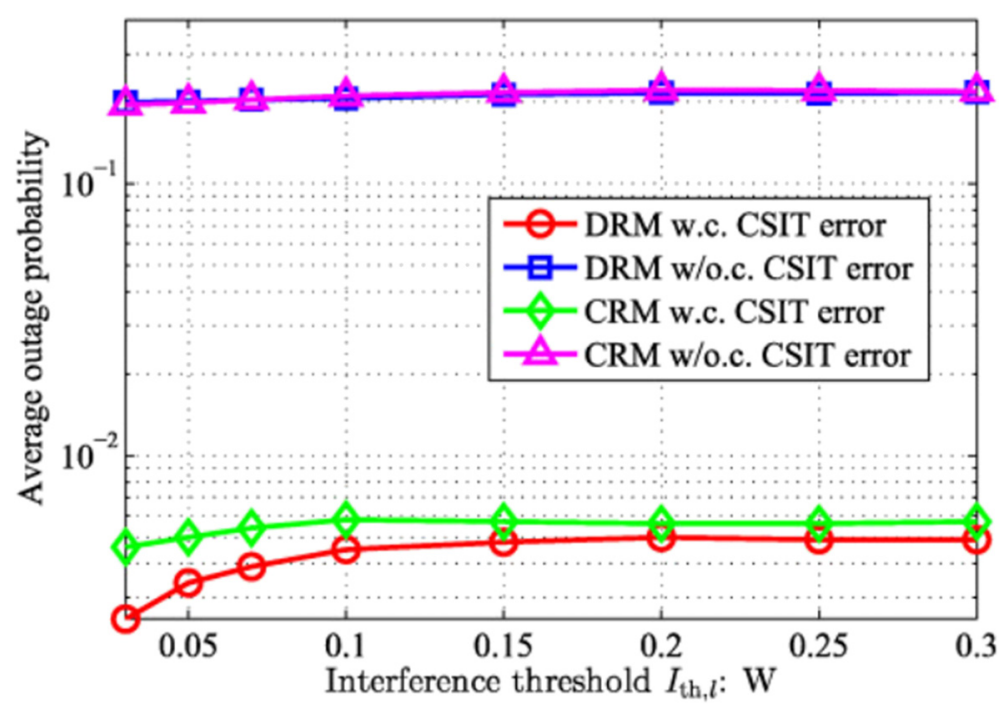

Figure 10 Average outage probability of cognitive destinations with different interference thresholds. The minimum required transmission SINR is set as $\gamma_{\text {th }}=150$, and the simulation results are averaged by 2,000 channel realizations. 


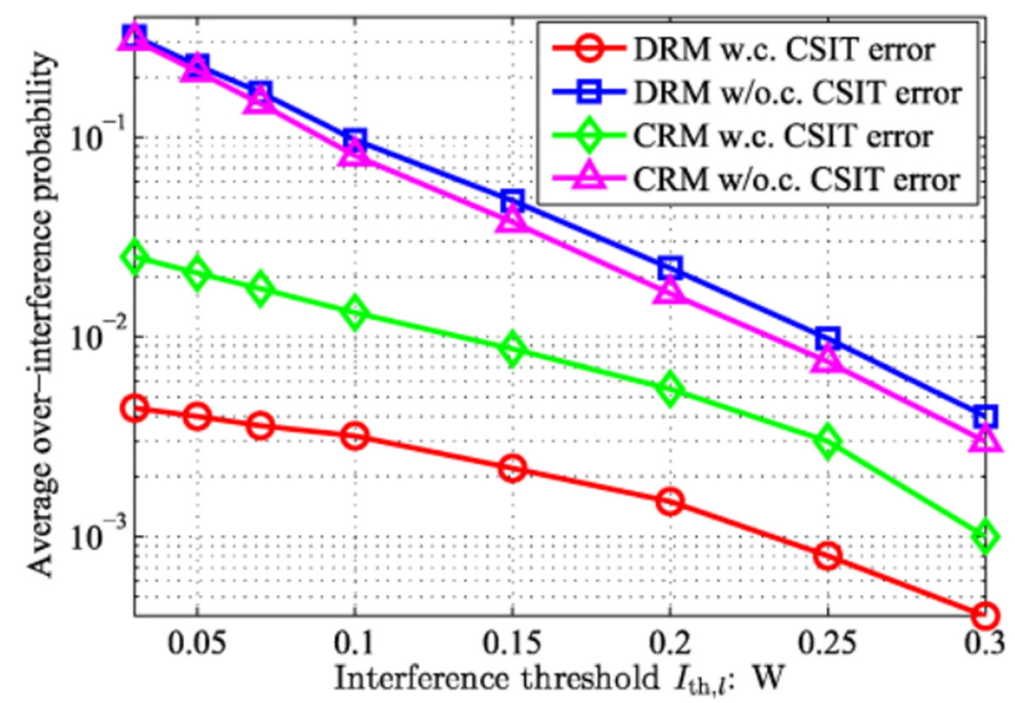

Figure 11 Average over-interference probability of primary users with different interference thresholds. In this figure, the minimum required transmission SINR is set as $\gamma_{\text {th }}=150$, and the simulation results are averaged by 2,000 channel realizations.

In case one where $K+L \geq N$, W can be rewritten as

$$
\mathbf{W}=\mathbf{H}[\mathbf{a}, \mathbf{B}]\left[\mathbf{f}, \mathbf{f}^{\perp}\right]^{H}=\mathbf{H a f}^{H}+\mathbf{H B}\left(\mathbf{f}^{\perp}\right)^{H}
$$

where $\mathbf{a} \in \mathbb{C}^{(K+L) \times 1}, \mathbf{B} \in \mathbb{C}^{(K+L) \times(N-1)}$.

A closer observation of problem $\mathcal{Q}$ reveals that the optimal $\mathbf{W}$ should minimize $\|\mathbf{W} \mathbf{f}\|^{2},\|\mathbf{W}\|^{2},\left|\mathbf{h}_{\mathrm{p}, l}^{H} \mathbf{W} \mathbf{f}\right|^{2}$, $\left\|\mathbf{h}_{\mathbf{p}, l}^{H} \mathbf{W}\right\|^{2},\left\|\mathbf{h}_{\mathrm{c}, k}^{H} \mathbf{W}\right\|^{2}$ and meanwhile maximize $\left|\mathbf{h}_{\mathrm{c}, k}^{H} \mathbf{W} \mathbf{f}\right|^{2}$. It is obvious that $\mathbf{B}$ does not affect $\left|\mathbf{h}_{\mathrm{c}, k}^{H} \mathbf{W f}\right|^{2},\|\mathbf{W} \mathbf{f}\|^{2}$, $\left|\mathbf{h}_{\mathbf{p}, l}^{H} \mathbf{W f}\right|^{2}$, and setting $\mathbf{B}=\mathbf{0}$ reduces $\|\mathbf{W}\|^{2},\left\|\mathbf{h}_{\mathrm{p}, l}^{H} \mathbf{W}\right\|^{2}$, $\left\|\mathbf{h}_{\mathrm{c}, k}^{H} \mathbf{W}\right\|^{2}$. As a result, the optimal $\mathbf{W}$ can be written as

$$
\mathbf{W}=\mathbf{H a f}^{H}=\mathbf{w f}^{H} .
$$

In case two where $K+L<N$, $\mathbf{W}$ can be rewritten as

$$
\begin{aligned}
\mathbf{W} & =\left[\mathbf{H}, \mathbf{H}^{\perp}\right]\left[\begin{array}{ll}
\mathbf{a} & \mathbf{B} \\
\mathbf{c} & \mathbf{D}
\end{array}\right]\left[\mathbf{f}, \mathbf{f}^{\perp}\right]^{H} \\
& =\mathbf{H a f} \mathbf{f}^{H}+\mathbf{H B}\left(\mathbf{f}^{\perp}\right)^{H}+\mathbf{H}^{\perp} \mathbf{c f}^{H}+\mathbf{H}^{\perp} \mathbf{D}\left(\mathbf{f}^{\perp}\right)^{H}
\end{aligned}
$$

where $\mathbf{c} \in \mathbb{C}^{(N-K-L) \times 1}, \mathbf{D} \in \mathbb{C}^{(N-K-L) \times(N-1)}$.

Similarly, it can be observed that $\mathbf{c}$ and $\mathbf{D}$ does not affect $\left|\mathbf{h}_{\mathrm{c}, k}^{H} \mathbf{W} \mathbf{f}\right|^{2},\left|\mathbf{h}_{\mathrm{p}, l}^{H} \mathbf{W} \mathbf{f}\right|^{2},\left\|\mathbf{h}_{\mathrm{p}, l}^{H} \mathbf{W}\right\|^{2},\left\|\mathbf{h}_{\mathrm{c}, k}^{H} \mathbf{W}\right\|^{2}$, and setting them as zero will reduce $\|\mathbf{W} \mathbf{f}\|^{2}$ and $\|\mathbf{W}\|^{2}$. Hence, the optimal $\mathbf{W}$ can be also written as

$$
\mathbf{W}=\mathbf{H a f}^{H}=\mathbf{w f}^{H} .
$$

In conclusion, the optimal structure of the forwarding matrix $\mathbf{W}$ in Lemma 1 is acquired.

\section{Competing interests}

The authors declare that they have no competing interests.

\section{Acknowledgements}

This work is supported by National Natural Science Foundation of China (61471059), the 863 Project No.2014AA01A701, fundamental Research Funds for the Central Universities (2014ZD03-01), Special Youth Science Foundation of Jiangxi (20133ACB21007).

Received: 8 August 2014 Accepted: 7 January 2015

Published online: 31 January 2015

References

1. 3GPP, Improvement of the Multimedia Broadcast Multicast Service (MBMS) in UTRAN, 3GPP TR 25.905 (2007)

2. GPP2, CDMA2000 High Rate Broadcast-Multicast Packet Data Air Interface Specification, 2GPP2 Std. C.S0054, Rev. 1.0 (2005)

3. IEEE, Air Interface for Fixed and Mobile Broadband Wireless Access Systems Amendment2: Physical and Medium Access Control Layers for Combined Fixed and Mobile Operation in Licensed Bands and Corrigendum 1. IEEE Std 802. 16e (2005)

4. A Abdelkader, M Pesavento, AB Gershman. IEEE International. Workshop Compun. Adv. Multi-Sensor Adaptive Process. San Juan, (2011), pp. 225-228

5. N Bornhorst, M Pesavento, AB Gershman, Distributed beamforming for multi-group multicasting relay networks. IEEE Trans. Signal Process. 60(1), 221-232 (2012)

6. V Havary-Nassab, S Shahbazpanahi, A Grami, Joint receive-transmit beamforming for multi-antenna relaying schemes. IEEE Trans. Signal Process. 58(9), 4966-4972 (2010)

7. M Dong, B Liang, in IEEE International Workshop on Compun. Adv. Multi-Sensor Adaptive Process., St. Martin. Multicast Relay Beamforming through Dual Approach, (2013), pp. 492-495

8. A Goldsmith, SA Jafar, I Maric, I Maric, S Srinivasa, Breaking spectrum gridlock with cognitive radios: an information theoretic perspective. Proc. IEEE. 97(5), 894-914 (2009)

9. L Zhang, YC Liang, Y Xin, HV Poor, Robust cognitive beamforming with partial channel state information. IEEE Trans. Wireless Commun. 8(8), 4143-4153 (2009)

10. R Zhang, Y Liang, S Cui, Dynamic resource allocation in cognitive radio networks. IEEE Signal Process Mag. 27(3), 102-114 (2010) 
11. SH Song, MO Hasna, KB Letaief, Prior zero forcing for cognitive relaying. IEEE Trans. Wireless Commun. 12(2), 938-947 (2013)

12. G Zheng, S Song, K Wong, B Ottersten, Cooperative cognitive networks: optimal, distributed and low-complexity algorithms. IEEE Trans. Signal Process. 61(11), 2778-2790 (2013)

13. AH Phan, HD Toan, HH Kha, DT Ngo, in IEEE Int. Conf. Commun. Electron., Nha Trang. A reverse convex programming for beamforming in cognitive multicast transmission, (2010), pp. 211-215

14. M Beko, Efficient beamforming in cognitive radio multicast transmission. IEEE Trans. Wirelesss Commun. 11(11), 4108-4117 (2012)

15. AH Phan, HD Tuan, HH Kha, DT Ngo, Nonsmooth optimization for efficient beamforming in cognitive radio multicast transmission. IEEE Trans. Signal Process. 60(6), 2941-2951 (2012)

16. KWang, T Chang, W Ma, AM So, C Chi, in IEEE ICASSP, Prague. Probabilistic SINR constrained robust transmit beamforming A Bernsteintype inequality based conservative approach, (2011), pp. 3080-3083

17. C Shen, TH Chang, KY Wang, Z Qiu, CT Chi, in IEEE Global Conference on Communications, Anaheim, CA. Chance-constrained robust beamforming for multi-cell coordinated downlink, (2012). pp. 4957-4962

18. P Marsch, G Fettweis, Coordinated Multi-Point in Mobile Communications. (Cambridge University Press, 2011)

19. I Bechar, A Bernstein-type Inequality for Stochastic Processes of Quadratic Forms of Gaussian Variables,. ArXiv e-prints (2009). arxiv.org/pdf/0909. 3595.pdf

20. E Karipidis, ND Sidiropoulos, ZQ Luo, Quality of service and max-min fair transmit beamforming to multiple cochannel multicast groups. IEEE Trans. Signal Process. 56(3), 1268-1279 (2008)

21. X Liu, H Li, H Wang, in IEEE International Conference on Communications and Networking in China, Guilin. Probability constrained robust multicast beamforming in cognitive radio network, (2013), pp. 708-712

22. Z Luo, W Ma, A So, Y Ye, S Zhang, Semidefinite relaxation of quadratic optimization problems. IEEE Signal Process. Mag. 27(3), 20-34 (2010)

\section{Submit your manuscript to a SpringerOpen ${ }^{\circ}$ journal and benefit from:}

- Convenient online submission

- Rigorous peer review

- Immediate publication on acceptance

- Open access: articles freely available online

- High visibility within the field

- Retaining the copyright to your article 